\title{
Measurements of turbulent diffusion in uniformly sheared flow
}

\author{
Christina Vanderwel ${ }^{1}$ and Stavros Tavoularis $^{1} \dagger$ \\ ${ }^{1}$ Department of Mechanical Engineering, University of Ottawa, Ottawa, ON K0A 3H0, Canada
}

(Received 12 July 2014 - Author's Accepted Version - doi: 10.1017/jfm.2014.406)

The diffusion of a plume of dye in uniformly sheared turbulent flow in a water tunnel was investigated using simultaneous stereoscopic particle image velocimetry and planar laserinduced fluorescence. Maps of the mean concentration and the turbulent concentration fluxes in planes normal to the plume axis were constructed, from which all components of the second-order turbulent diffusivity tensor were determined for the first time. Good agreement between the corresponding apparent and true diffusivities was observed. The turbulent diffusivity tensor was found to have strong off-diagonal components, whereas the streamwise component appeared to be counter-gradient. The different terms in the advection-diffusion equation were estimated from the measurements and their relative significances were discussed. All observed phenomena were explained by physical arguments and the results were compared to previous ones.

Key words: Turbulent diffusion, Homogeneous turbulence, Mixing and dispersion

\section{Introduction}

Turbulent diffusion is the process of spreading and mixing of admixtures by turbulent motions. It is the essential mechanism that drives dispersal of pollutants in the environment, mixing in industrial processes, and chemical reactions in diverse systems. Although turbulent diffusion has been studied extensively for a long time, its analysis for engineering purposes is still based on crude models and is mostly concerned with the prediction of the mean concentration of the admixture (Roberts \& Webster 2002).

The exact governing equation for the mean concentration $\bar{C}$ of an admixture released passively in a turbulent flow is the Reynolds-averaged advection-diffusion equation

$$
\frac{\partial \bar{C}}{\partial t}+\underbrace{\overline{U_{i} \frac{\partial \bar{C}}{\partial x_{i}}}}_{\text {advection }}=\underbrace{\gamma \frac{\partial^{2} \bar{C}}{\partial x_{i} \partial x_{i}}}_{\text {molecular diffusion }}+\underbrace{\frac{\partial\left(-\overline{c u_{i}}\right)}{\partial x_{i}}}_{\text {turbulent diffusion }},
$$

where $\overline{U_{i}}$ is the mean velocity vector, $\gamma$ is the molecular diffusivity, and $\overline{c u_{i}}$ is the concentration-velocity covariance or turbulent mass flux vector. Even if the mean velocity field were specified, this equation would be open in $\bar{C}$. One type of closure is possible by the use of a model for the turbulent mass flux vector in terms of $\bar{C}$. The most commonly used model is the first-order gradient transport model (Arya 1999)

$$
-\overline{c u_{i}}=D_{i j} \frac{\partial \bar{C}}{\partial x_{j}}
$$

$\dagger$ Email address for correspondence: stavros.tavoularis@uottawa.ca 
where $D_{i j}$ is the turbulent, or "eddy", diffusivity tensor, which has nine components, the values of which depend on the turbulent field. The gradient transport model is based on the assumptions that the macroscopic characteristic lengthscale of the scalar is much greater than that of the transporting mechanism (i.e., the turbulence) and that the flow properties are approximately homogeneous over the turbulence lengthscale (Corrsin 1974). Despite the fact that these assumptions are not satisfied in most cases of interest, the gradient transport model is known to provide fairly accurate predictions in a variety of situations (Sreenivasan et al. 1982). Application of (1.2) requires empirical knowledge of all nine components of $D_{i j}$. Nevertheless, measurements of these components are quite scarce, due to the difficulty of measuring simultaneously the local magnitudes and directions of both the mean concentration gradient and the turbulent mass flux vector. Theoretical expressions relating $D_{i j}$ to turbulent characteristics have also been suggested; these will be discussed in following sections. Some models simplify the turbulent diffusivity tensor by assuming that it is diagonal or isotropic, but the use of such simplified models in shear flows is known to introduce additional errors.

The objective of the present work is to study turbulent diffusion in a shear flow generated in the laboratory and specifically to measure all components of the turbulent diffusivity tensor in this flow. The scalar property that has been measured is the concentration of dye in a plume released nearly passively from a thin tube in uniformly sheared flow (USF). The statistical properties of the velocity and scalar concentration fields were measured simultaneously on cross-sectional planes using stereoscopic particle image velocimetry (SPIV) and planar laser-induced fluorescence (PLIF). Thus, two-dimensional maps of the turbulent velocities and scalar concentration were obtained, as well as maps of the three concentration-velocity covariances; from these maps, the values of all components of the turbulent diffusivity tensor were determined directly by fitting (1.2) to the data. This is the first time all nine components of the turbulent diffusivity tensor have been determined together experimentally. This article will also present comparisons of the experimental results to theoretical estimates of the turbulent diffusivities.

\section{Literature on turbulent diffusion}

The study of turbulent diffusion originated with the work of Taylor (1921), who considered the one-dimensional motion of an individual fluid particle released from a fixed point in stationary isotropic turbulent flow, neglecting molecular diffusion. He demonstrated that the variance of an ensemble of particle displacements following time $t$ from their release should depend only on the turbulence properties as

$$
\overline{X^{2}}(t)=2 \overline{v^{2}} \int_{0}^{t}(t-\xi) R(\xi) d \xi,
$$

where $v$ represents the Lagrangian velocity fluctuations and $R(\xi)$ is the Lagrangian correlation coefficient. Taylor further identified two regimes of diffusion which would occur in the limits of short $t$ and long $t$, known as the turbulent convection and turbulent diffusion regimes (Anand \& Pope 1985). In these regimes, (2.1) would be simplified as, respectively,

$$
\begin{array}{r}
\overline{X^{2}}(t) \approx \overline{v^{2}} t^{2}, \text { for } t \ll \mathcal{T}, \\
\overline{X^{2}}(t) \approx 2 \overline{v^{2}} \mathcal{T} t \text {, for } \mathcal{T} \ll t,
\end{array}
$$


where $\mathcal{T}$ is the Lagrangian integral timescale of the turbulence. It follows that the rate of dispersion, defined as

$$
\frac{1}{2} \frac{\mathrm{d} \overline{X^{2}}(t)}{\mathrm{d} t}=\overline{v^{2}} \int_{0}^{t} R(\xi) d \xi,
$$

has the following limits for the near- and far-field regimes

$$
\begin{gathered}
\frac{1}{2} \frac{\mathrm{d} \overline{X^{2}}(t)}{\mathrm{d} t} \approx \overline{v^{2}} t, \text { for } t \ll \mathcal{T}, \\
\frac{1}{2} \frac{\mathrm{d} \overline{X^{2}}(t)}{\mathrm{d} t} \approx \overline{v^{2}} \mathcal{T}, \text { for } \mathcal{T} \ll t .
\end{gathered}
$$

This implies that the rate of dispersion would be zero at the moment of particle release, would initially increase linearly with dispersion time, and would eventually reach an asymptote which would depend only on the Lagrangian integral timescale of the turbulence and the variance of the Lagrangian velocity fluctuations.

The theoretical analysis of three-dimensional turbulent diffusion was first presented by Batchelor (1949), who defined a three-dimensional diffusion coefficient tensor $\frac{1}{2} \mathrm{~d} \overline{X_{i} X_{j}} / \mathrm{d} t$ in terms of the mean Lagrangian displacement tensor $\overline{X_{i} X_{j}}(t)$ of a particle transported by homogeneous turbulence. He further demonstrated that, in homogeneous, non-sheared turbulence and for a Gaussian particle displacement distribution, $\frac{1}{2} \mathrm{~d} \overline{X_{i} X_{j}} / \mathrm{d} t$ would be equal to the turbulent diffusivity tensor $D_{i j}$, as defined in (1.2). Batchelor also showed that, in isotropic turbulence, $D_{i j}$ would be proportional to the identity tensor (Kronecker's delta); as in Taylor's theory, the magnitudes of the components of $D_{i j}$ would increase with dispersion time and would eventually reach asymptotes that depend only on the Lagrangian integral timescales of the turbulence $\mathcal{T}_{i j}$ and the intensities of the Lagrangian velocity fluctuations.

Riley \& Corrsin (1974) expanded Batchelor's analysis for homogeneous turbulent shear flow and noted that the normal diffusivities were unequal and that some of the crossdiffusivities were not zero. As with the theories of Taylor and Batchelor, the magnitudes of the turbulent diffusivities were shown to depend on the Lagrangian integral timescales of the turbulence $\mathcal{T}_{i j}$ and the intensities of the Lagrangian velocity fluctuations. Corrsin (1974) suggested that the asymptotic values of the turbulent diffusivities could be estimated from Eulerian properties, as surrogates for their Lagrangian counterparts, for example as $D_{22} \approx u_{2}^{\prime 2} T_{11}$. Additional theoretical arguments have been made by Tavoularis \& Corrsin (1985), Rogers et al. (1989) and Younis et al. (2005) to derive asymptotic expressions for the turbulent diffusivity tensor components in shear flows. These theories will be revisited in a later section.

A classical approach for measuring turbulent diffusion in the laboratory or in the environment is to relate the rate of growth of puffs and plumes generated in the turbulent flow to an apparent turbulent diffusivity. The plume generated by a continuously emitting source may be considered as the result of superposition of clouds emitted successively by an instantaneous source. For a plume of particles emitted from a point source in a turbulent flow with a uniform mean velocity $U_{1}$ in the $x_{1}$ direction, the apparent diffusivities in the two normal directions $x_{2}$ and $x_{3}$ are defined as

$$
K_{2}=\frac{U_{1}}{2} \frac{\mathrm{d} \sigma_{2}^{2}}{\mathrm{~d} x_{1}}, \quad K_{3}=\frac{U_{1}}{2} \frac{\mathrm{d} \sigma_{3}^{2}}{\mathrm{~d} x_{1}}
$$

where $\sigma_{2}$ and $\sigma_{3}$ are the corresponding characteristic plume widths. If, and only if, the streamwise dispersion were negligible, the apparent plume diffusivities $K_{2}$ and $K_{3}$ would 
be equivalent to the diffusion coefficients $\frac{1}{2} \mathrm{~d} \overline{X_{2}^{2}} / \mathrm{d} t$ and $\frac{1}{2} \mathrm{~d} \overline{X_{3}^{2}} / \mathrm{d} t$ (Arya 1999), and thus to $D_{22}$ and $D_{33}$.

Further assuming that the two apparent turbulent diffusivities are uniform in space and equal to each other $\left(K_{2}=K_{3}=K\right)$ leads to the well known Gaussian plume formula (Arya 1999) for the mean concentration of a continuous plume in uniform mean flow

$$
\bar{C}=\frac{Q}{4 \pi K x_{1}} \exp \left[-U_{1}\left(\frac{x_{2}^{2}+x_{3}^{2}}{4 K x_{1}}\right)\right] .
$$

According to this solution, the maximum concentration of the plume would decay as $x_{1}^{-1}$ and the plume width would grow as $x_{1}^{0.5}$.

Examples of investigations of turbulent diffusion in the environment include early observations of anti-aircraft shell-bursts (Roberts 1923; Sutton 1932) and plumes of lycopodium spores released in the atmosphere (Hay \& Pasquill 1959) and dyes released in a lake (Csanady 1963). Examples of relevant laboratory studies include investigations of diffusion behind a line source in grid turbulence (Warhaft 1984; Anand \& Pope 1985; Stapountzis et al. 1986), a line source in uniformly sheared flow (Tavoularis \& Corrsin 1981; Karnik \& Tavoularis 1989), a line source in channel flow (Lepore \& Mydlarski 2011), a point source in uniformly sheared flow (Nakamura et al. 1986), and a point source in channel flows (Webster et al. 2003; Rahman \& Webster 2005). In these actual turbulent flows, the turbulent kinetic energy and lengthscale would generally evolve downstream and so would the diffusivities of superimposed scalar plumes. As a result, (2.8) would not be applicable and the plume growth rate would be expected to deviate from the previously mentioned power law. Experimental studies of plumes in grid turbulence and shear flows have found that the plume widths followed power laws but with powers different from the theoretical value of 0.5 . This discrepancy between the simplified solutions and actual turbulent flows highlights the need for further studies of turbulent diffusion and refined turbulent diffusion models.

Individual components of the turbulent diffusivity tensor, as defined by (1.2), can be calculated from measurements of corresponding mean scalar derivatives and velocityscalar covariances. Most experimental studies that reported such results have taken them at locations where the scalar gradient was nearly aligned with one of the physical mean flow axes. Tavoularis \& Corrsin (1981) examined a case in which a uniform mean scalar gradient was superimposed on USF, with both gradients in the $x_{2}$ direction, and measured $D_{12} / D_{22} \approx-2.2$. Tavoularis \& Corrsin (1985) examined a variant of this case, in which the mean scalar gradient was in the $x_{3}$ direction, and found $D_{33} / D_{22} \approx 1.6$. Karnik \& Tavoularis (1989) studied the plume of a line source in USF, where the mean scalar gradient was nearly aligned with the $x_{2}$ direction and reported that $D_{12} / D_{22} \approx-2.0$ and $D_{22} /\left(u_{2}^{\prime} L_{11,1}\right) \approx 0.1$.

The literature includes several measurements of scalar turbulent diffusivities in various inhomogeneous shear flows, as for example in turbulent jets (Lemoine et al. 1996; Borg et al. 2001; Chang \& Cowen 2002). In general, such diffusivities were determined from mean scalar and turbulent scalar flux profiles in a single direction, with no consideration given to the possible three-dimensionality of the mean scalar field and the turbulence inhomogeneity. In consequence, it seems plausible that such scalar diffusivities would depend on the orientation, as well as location, of the data profiles used and, indeed, Borg et al. (2001) found drastically different diffusivity values in the radial and axial directions in a jet. Consequently, scalar diffusivity measurements in inhomogeneous shear flows are deemed to be specific to particular flow geometries and experimental conditions and should not be considered for general use in other turbulent flows. 


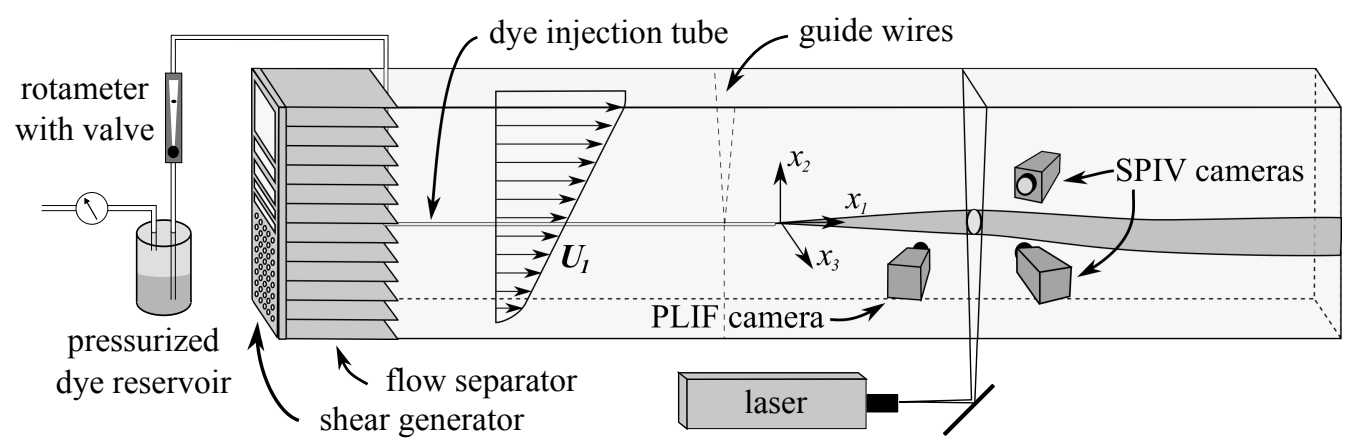

Figure 1: Sketch of the experimental apparatus and main instrumentation in the water tunnel test section.

A review of past literature has identified no measurements of all components of the turbulent diffusivity tensor that were taken at once in the same flow. The present study aims at filling this gap in the literature.

\section{Apparatus and experimental procedures}

The experiments were conducted in a free-surface, recirculating water tunnel, having a test section with a width of $0.53 \mathrm{~m}$, a length of about $4 \mathrm{~m}$, and filled to a depth of $0.46 \mathrm{~m}$ (figure 1). USF was generated by a perforated plate of varying solidity ("shear generator"), inserted at the entrance to the test section and followed by an array of parallel plates spaced by a distance $L=25.4 \mathrm{~mm}$ ("flow separator").

A neutrally buoyant aqueous solution of Rhodamine $6 \mathrm{G}$ fluorescent dye with a concentration $C_{S}=0.3 \mathrm{mg} / \ell$ was injected into the flow through a fine tube having a tip with an inner diameter of $1.83 \mathrm{~mm}$ and a wall thickness of $0.15 \mathrm{~mm}$. To minimize its disturbance to the flow, the tube was inserted in the stream through the flow separator and was aligned with the flow section centreline so that the dye was discharged at approximately $2 \mathrm{~m}$ downstream of the flow separator, where the turbulence structure of the USF was fully developed. The injection tube was tethered by $50 \mu \mathrm{m}$ thick guide wires, and was free of any movement or vibrations. The dye solution was contained in a reservoir that was pressurized by compressed air through a pressure regulator. The dye solution flow rate through the injection tube was measured by a rotameter and adjusted with a built-in needle valve so that injection created the least possible flow disturbance. The optimal flow rate was found to be $Q=0.97 \pm 0.05 \mathrm{~m} \ell / \mathrm{s}$ (see appendix A). The dye molecular diffusivity was taken to be $\gamma=(4.0 \pm 0.3) \times 10^{-4} \mathrm{~mm}^{2} / \mathrm{s}$ (Gendron et al. 2008), which corresponds to a Schmidt number $S c \equiv \nu / \gamma=2500 \pm 300$. Rhodamine $6 \mathrm{G}$ has an absorption peak at $525 \mathrm{~nm}$, which is close to the emission wavelength of the Nd:YAG laser $(532 \mathrm{~nm}$ ); it also has an emission peak at $554 \mathrm{~nm}$ (Würth et al. 2012), which is sufficiently different from the laser emission peak for this substance to be well suited for PLIF measurements. The camera used to measure the fluorescence was fitted with an optical longpass filter with a sharp cutoff at $540 \mathrm{~nm}$, so as to be sensitive only to the fluorescence emitted by the dye and to block essentially all incident light produced by the laser and ambient light. Dye solutions were prepared by mixing Rhodamine 6G powder (A\&C American Chemicals Ltd., CAS: 989-38-8, Montreal, Canada) with distilled water.

Velocity and concentration measurements were taken simultaneously in cross-sections of the flow normal to the streamwise direction, that were illuminated by a light sheet cre- 
ated from the output of a Nd:YAG pulsed laser. Velocity measurements were performed using a two-camera SPIV system (LaVision GmbH, FlowMaster Stereo-PIV, Göttingen, Germany). Concentration measurements were obtained using a third camera (PCO AG, pco.edge, Kelheim, Germany), which had a capacity of 5.5 MP, a pixel depth of 16 bits, and was synchronized with the SPIV system and the laser pulse triggering circuit. All cameras were fitted with Scheimpflug adapters, so that the entire measurement plane would be in focus, although viewed by the camera at an inclination. Liquid prisms were used to eliminate horizontal astigmatism that would have been introduced in the images if the cameras had viewed the flow at an inclination of $45^{\circ}$ with respect to the glass wall (Prasad \& Jensen 1995; Adrian \& Westerweel 2011). The field of view of the measurements was rectangular with an approximately $120 \mathrm{~mm}$ vertical side and a $200 \mathrm{~mm}$ horizontal side. The fields of view of the three cameras were carefully aligned using a twosided calibration target (LaVision GmbH, calibration plate \#22), which was mounted in the image plane before the experiment. Images of the calibration plate taken with each camera were then processed by the software provided by the manufacturer (LaVision $\mathrm{GmbH}, \mathrm{DaVis}$ 7.2) to create image mapping functions that ensured that the three images were coincident. The mapping functions were then fine-tuned using a self-calibration process applied to images of particles illuminated by the laser sheet taken with each camera, in order to correct for any misalignment between the laser sheet and the calibration target. Following this calibration, the average misalignment of the cameras was estimated to be 3.4 pixels, which corresponds to approximately $0.2 \mathrm{~mm}$ in the field of view.

Measurements were obtained in five cross-sectional planes, located at dimensionless distances from the source equal to $x_{1} / L=5,12,20,28$, and 35 . The in-plane spatial resolutions of the measured velocity and concentration fields were, respectively, one vector per $1.15 \mathrm{~mm} \times 1.15 \mathrm{~mm}$ flow area and one scalar sample per $0.05 \mathrm{~mm} \times 0.05 \mathrm{~mm}$ area. The cross-sectional profile of the laser intensity had approximately the shape of a Gaussian distribution with a standard deviation of $1 \mathrm{~mm}$, which, considering the $45^{\circ}$ viewing angle, corresponds to a resolution along the line of sight of the camera of approximately $2.8 \mathrm{~mm}$. The time delay between pulses for each SPIV measurement was $1500 \mu \mathrm{s}$. During the interval between the two pulses, a particle travelling with the centreline flow velocity of $0.18 \mathrm{~m} / \mathrm{s}$ would be displaced by a streamwise distance of $0.27 \mathrm{~mm}$, which satisfies well the SPIV requirement of being much smaller than the laser sheet thickness. Pulseto-pulse laser power fluctuations had a standard deviation of approximately $3 \%$, which was deemed to be acceptable for PLIF measurements. Concentration and velocity measurements were sampled at a rate of $2 \mathrm{~Hz}$. Ensemble averages were calculated from 500 samples acquired at each of the positions $x_{1} / L=5,12$, and 20, and from 1000 samples at $x_{1} / L=28$ and 35. Differences between plume widths computed by averaging 500 samples at $x_{1} / L=28$ and 35 and those computed by averaging 1000 samples had a standard deviation of $3 \%$, which is deemed to be sufficiently small for considering averages of 500 samples to be essentially convergent.

The concentration $C$ of dye measured by each diode (i.e., each pixel) of the camera was determined as a linear function of the radiant power flux emitted by the dye ("the fluorescence") in the plane of the laser sheet. The coefficients in this function were determined independently for each pixel of the camera from a calibration measurement in a small calibration tank, placed in situ and filled with a dye solution having a uniform concentration $C_{\text {cal }}=0.075 \mathrm{mg} / \mathrm{L}$. Pixel-by-pixel calibration accounted for the effects of spatial variation in the laser sheet and optical components, lens vignette, and any pixel-to-pixel offsets or gain variations in the camera.

Concentration measurement errors and uncertainties in slender plume using PLIF have been the subject of a separate manuscript (Vanderwel \& Tavoularis 2014a). In this work 
we quantified several hitherto disregarded sources of error, which may potentially contaminate significantly fluorescent dye concentration measurements by PLIF. One source of error is secondary fluorescence, which is the result of the absorption and re-emission of primary fluorescence by dye both within and outside the laser sheet. Secondary fluorescence was at least one, and mostly two or more, orders of magnitude weaker than the primary fluorescence emitted by the dye excited by the laser sheet, but its contributions to the recorded radiation power flux were sufficient to bias both the calibration and the instantaneous concentration maps, if they had remained unaccounted for. We derived and applied novel methods to remove the effects of secondary fluorescence from the camera calibration results and the instantaneous concentration maps and also devised an effective procedure to identify the boundaries of the in-sheet dye regions in the instantaneous concentration maps. We further demonstrated that secondary fluorescence had a very weak effect on the measurement of concentration fluctuations and so we are confident that the bulk of results presented in this article are insensitive to this undesirable input. Another source of uncertainty, which applies to PLIF measurements in all flows but is exacerbated in very slender plumes, is the fact that dye concentration is not uniform along the entire thickness of the laser sheet, but a part of the fluid across this sheet may sometimes contain little or no dye. This effect would bias negatively instantaneous concentration measurements that were based on calibration of the camera in a fluid with a uniform concentration. We have analyzed this effect and demonstrated that such bias would be negligible for distances from the source beyond $x_{1} / L \approx 20$. We also found that both secondary fluorescence and dye-non-uniformity had negligible effects on the values of the plume width, lengthscales and turbulent diffusivities reported in this article.

\section{Results}

\subsection{The velocity field}

The USF and its turbulence structure in the same facility have been documented previously by Vanderwel \& Tavoularis (2011), to be referred to as VT in the remainder of this article. New velocity measurements were taken on transverse planes at several streamwise locations in the plume. The origin of coordinates is set at the centre of the injection tube exit face (figure 1), and the term "centreline" refers to the $x_{1}$ axis. The mean centreline velocity $U_{c}$ and the mean velocity gradient $\mathrm{d} \bar{U}_{1} / \mathrm{d} x_{2}$ in the undisturbed flow were essentially constant. Vertical profiles of the mean velocity and the velocity standard deviation in the $\left(x_{1}, x_{2}\right)$ plane are presented in figure 2. Near the exit of the dye injector, the mean and turbulent velocities were slightly disturbed, however, further downstream the mean velocity profile became nearly linear and the turbulence became nearly transversely homogeneous, in agreement with the VT results and those in other USF studies. Specifically, for $x_{1} / L=20,28$, and 35 , measurements of the mean velocity deviated from the fitted lines by less than $2.5 \%$ in the field of view and measurements of the r.m.s. turbulent velocity components had a standard deviation of approximately $5 \%$; these deviations are in line with the observations of VT in fully developed USF and are sufficiently small for the flow to be considered as nearly homogeneous. The streamwise evolutions of the normalized r.m.s. turbulent velocities are plotted in figure 3, together with those reported by VT at a slightly lower water tunnel speed. It is noted that the uncertainty of the VT measurements was measurably lower than the present one, because VT took averages over long time histories of laser Doppler velocimetry signals. Near the exit of the dye injector, the turbulent velocities appear to be slightly stronger than the VT values, however, at the other locations the corresponding measurements 

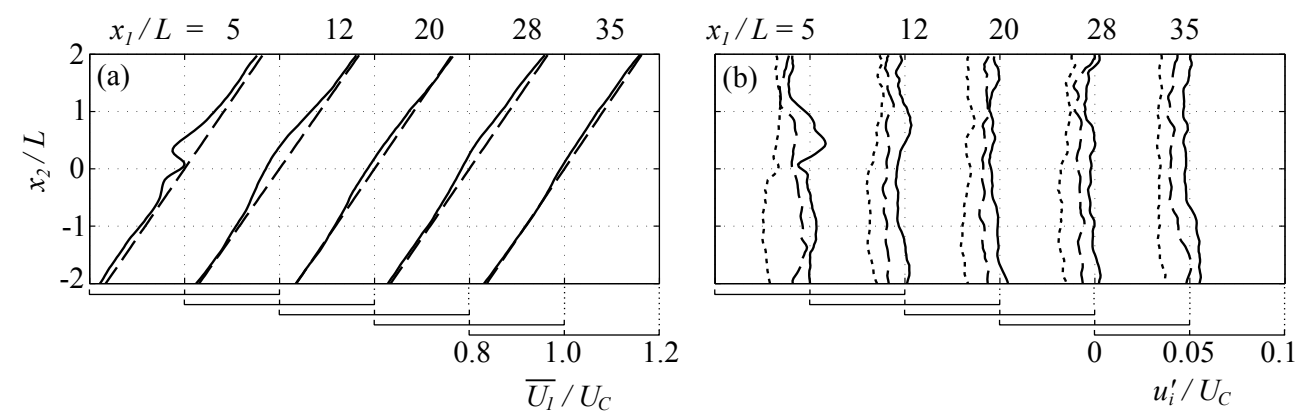

Figure 2: Transverse profiles of (a) the mean streamwise velocity in the plume (solid line), together with the linear function $U_{c}+x_{2} \mathrm{~d} \bar{U}_{1} / \mathrm{d} x_{2}$ (dashed line) and (b) the r.m.s. turbulent velocity components in the plume $\left(u_{1}^{\prime} / U_{C}\right.$ : solid line, $u_{2}^{\prime} / U_{C}$ : dotted line, and $u_{3}^{\prime} / U_{C}$ : dashed line); $x_{3} / L=0$.

$$
\begin{aligned}
U_{c} & =0.18 \mathrm{~m} / \mathrm{s} \\
k & =86 \mathrm{~mm}^{2} \mathrm{~s}^{-2} \\
m_{11} & =0.12 \\
m_{33} & =-0.01 \\
L_{11,1} & =30.5 \mathrm{~mm} \\
\eta & =0.60 \mathrm{~mm} \\
S^{*} & =13.4 \\
\nu_{T} & =58 \mathrm{~mm}^{2} \mathrm{~s}^{-1}
\end{aligned}
$$

$$
\begin{aligned}
\mathrm{d} \bar{U}_{1} / \mathrm{d} x_{2} & =0.59 \mathrm{~s}^{-1} \\
\epsilon & =7.6 \mathrm{~mm}^{2} \mathrm{~s}^{-3} \\
m_{22} & =-0.12 \\
m_{12} & =-0.11 \\
\lambda_{11} & =17 \mathrm{~mm} \\
\eta_{B} & =0.012 \mathrm{~mm} \\
R_{\lambda_{11}} & =150
\end{aligned}
$$

Table 1: Measured or estimated mean and turbulence parameters at $x_{1} / L=35$.

were comparable. The average turbulent kinetic energy $k$ was essentially the same as that measured by VT, which justifies the use of the kinetic energy dissipation rate $\epsilon$ values reported by VT for the present flow as well. In USF, the turbulent kinetic energy grows exponentially from an effective origin far upstream of the plume origin. Table 1 summarizes parameters of interest at $x_{1} / L=35$. In this table, the turbulence anisotropies are defined as $m_{i j}=\overline{u_{i} u_{j}} / 2 k-\delta_{i j} / 3 ; L_{11,1}$ is the streamwise integral length scale; $\lambda_{11}$ is the streamwise Taylor microscale, estimated as $\lambda_{11} \simeq(24 \nu k / \epsilon)^{1 / 2}$ (De Souza et al. 1995); $\eta=\left(\nu^{3} / \epsilon\right)^{1 / 4}$ is the Kolmogorov microscale; $\eta_{B}=\eta / S c^{1 / 2}$ is the Batchelor microscale; $S^{*} \equiv(2 k / \epsilon)\left(\mathrm{d} \bar{U}_{1} / \mathrm{d} x_{2}\right)$ is the shear rate parameter; $R e_{\lambda_{11}}=u_{1}^{\prime} \lambda_{11} / \nu$ is the turbulence Reynolds number; and $\nu_{T} \equiv-\overline{u_{1} u_{2}} /\left(\mathrm{d} \bar{U}_{1} / \mathrm{d} x_{2}\right)$ is the turbulent viscosity, which grew to the reported value from a value that was $41 \%$ lower at $x_{1}=0$.

\subsection{Velocity integral lengthscales}

Theoretical models of turbulent diffusion make use of various integral lengthscales of the turbulent fluctuations. Previous measurements in USF have been mainly limited to the streamwise lengthscales of different velocity components, obtained from single-point laser Doppler or hot-wire time histories with the use of Taylor's approximation (e.g., Tavoularis \& Corrsin 1981; Vanderwel \& Tavoularis 2011). However, it is the transverse lengthscale of the transverse velocity, and the corresponding spanwise scale, that are relevant to transport and diffusion, and these scales are not easily measurable by laser Doppler and hot-wire anemometries. The current SPIV measurements provide an excellent opportunity to measure directly the transverse and spanwise velocity autocorrelation functions 


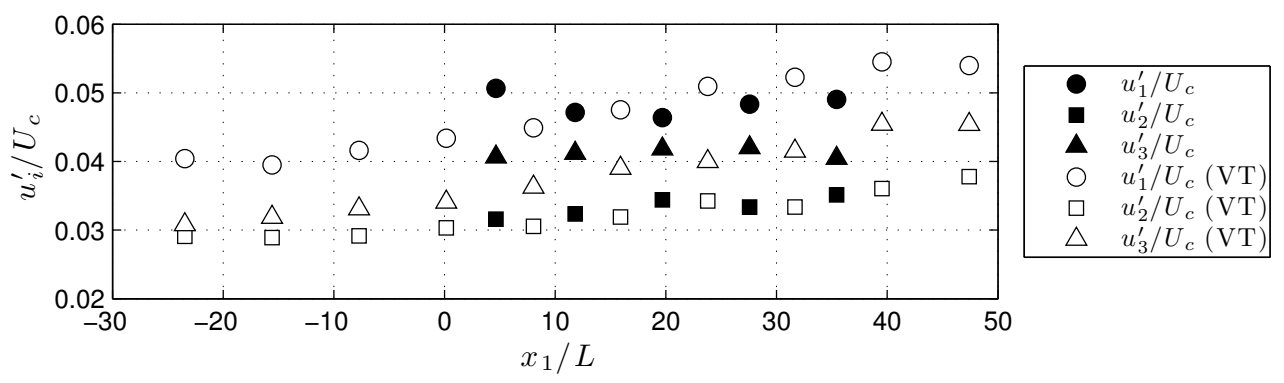

Figure 3: Streamwise development of the r.m.s. velocity fluctuations compared to LDV measurements from Vanderwel \& Tavoularis (2011).

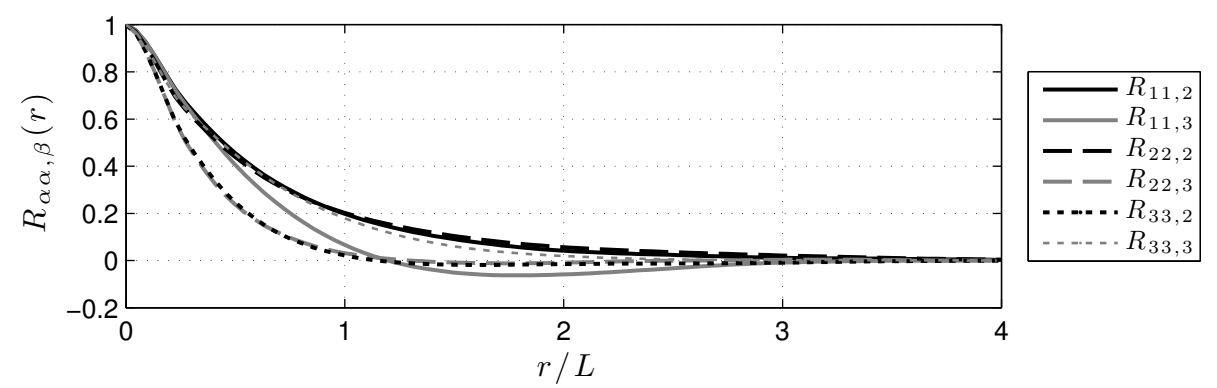

Figure 4: Measured autocorrelations functions on the $\left(x_{2}, x_{3}\right)$ plane, at $x_{1} / L=28$.

$\begin{array}{ccccccccc} & \frac{L_{11,2}}{L_{11,1}} & \frac{L_{11,3}}{L_{11,1}} & \frac{L_{22,1}}{L_{11,1}} & \frac{L_{22,2}}{L_{11,1}} & \frac{L_{22,3}}{L_{11,1}} & \frac{L_{33,1}}{L_{11,1}} & \frac{L_{33,2}}{L_{11,1}} & \frac{L_{33,3}}{L_{11,1}} \\ \text { SPIV } & 0.54 & 0.39 & - & 0.55 & 0.31 & - & 0.30 & 0.49 \\ \text { PPIV } & 0.51 & - & 0.58 & 0.60 & - & - & - & -\end{array}$

Table 2: Average ratios of the integral lengthscales.

and integral lengthscales. The measured components of the autocorrelation function tensor, defined as

$$
R_{\alpha \alpha, \beta}(r)=\frac{\overline{u_{\alpha}\left(x_{\beta}\right) u_{\alpha}\left(x_{\beta}+r\right)}}{\overline{u_{\alpha}^{2}\left(x_{\beta}\right)}} .
$$

are presented in figure 4 . These were computed from SPIV images on the $\left(x_{2}, x_{3}\right)$ plane. The corresponding integral lengthscales were calculated by integrating the corresponding autocorrelation functions up to their first zero-crossing (O'Neill et al. 2004). Additional lengthscales were calculated from planar PIV (PPIV) measurements in the $\left(x_{1}, x_{2}\right)$ plane. The integral lengthscales were found to grow downstream, however, within the reported range of the plume, they only changed by about $5-10 \%$. The average value of $L_{11,1}$ was about $1.2 L$, in agreement with the LDV measurements of VT. The average ratios of the measured integral lengthscales are summarised in table 2 . The values of these scales demonstrate a strong anisotropy of the turbulence in the energy containing range. 


\subsection{The concentration field}

Two representative instantaneous concentration maps are presented in figure $5 \mathrm{a}$, b; in one case, the blown-up part also shows the in-plane velocity vector map, which, as mentioned previously, had a spatial resolution that was much lower than that of the concentration. The maps clearly show the presence of mushroom-type patterns, which are evidence of horseshoe vortices in the USF (Vanderwel \& Tavoularis 2011). The plume was observed to meander significantly within the field of view (Vanderwel \& Tavoularis 2014b), with the result that large portions of the instantaneous maps had zero concentration, whereas the areas coincident with the dye plume had relatively high concentrations that often exceeded the peak time-averaged concentration on that plane by a factor of 20 . The intermittency of the local concentration can be measured by the intermittency factor $\gamma_{c}$, which is defined as the fraction of time during which the concentration was non-zero (Wilson et al. 1985). Close to the plume source, at $x_{1} / L=5$, the intermittency on the plume axis was moderate, as the peak value of $\gamma_{c}$ was approximately 0.4. However, for $x_{1} / L>12$, the peak $\gamma_{c}$ remained low, at approximately 0.23 ; in this range, the transverse profile of the intermittency factor was self-similar and had an essentially Gaussian shape (see figure 6a). Strong scalar intermittency is specific to the present plume, which is relatively slender and also contains a dye with an extremely small molecular diffusivity. These specific conditions differentiate the present configuration from thermal plumes in wind tunnels (e.g., Tavoularis \& Corrsin 1981; Karnik \& Tavoularis 1989; Lepore \& Mydlarski 2011).

The values at each pixel of all recorded instantaneous concentration maps were averaged to produce the corresponding mean concentration maps. It was found that, away from regions containing dyed water, the measured concentration was approximately $2.5 \times 10^{-4} C_{S}$, which was deemed to be the background concentration "noise" level. Each mean concentration map was fitted by a two-dimensional Gaussian function

$$
\frac{\bar{C}}{C_{S}}=A \exp \left[-\frac{\left(x_{2}-\mu_{2}\right)^{2}}{2 \sigma_{2}^{2}}-\frac{\left(x_{3}-\mu_{3}\right)^{2}}{2 \sigma_{3}^{2}}\right],
$$

where $A$ is a dimensionless amplitude, $\mu_{2}$ and $\mu_{3}$ are the transverse and spanwise coordinates of the mean plume axis, and $\sigma_{2}^{2}$ and $\sigma_{3}^{2}$ are the corresponding second central moments of the concentration distribution; all these parameters are functions of streamwise location $x_{1}$. The parameters $A, \mu_{2}, \mu_{3}, \sigma_{2}$ and $\sigma_{3}$ were determined using the MATLAB Curve Fitting Toolbox (MathWorks, Natick, MA, USA) as those that resulted in the best fit to the mean concentrations measured at each pixel. The curve fitting used an iterative least-squares algorithm (Trust-Region Reflective Newton) starting from the initial guesses $A=0.01, \sigma_{2}=10, \sigma_{3}=10, \mu_{2}=0$, and $\mu_{3}=0$ and restricting the parameters $A$, $\sigma_{2}$, and $\sigma_{3}$ to positive values. A representative mean concentration map $\bar{C} / C_{S}$ is provided in figure $5 \mathrm{c}$, together with isocontours of the fitted two-dimensional Gaussian function. Figure $6 \mathrm{~b}$ shows normalized transverse profiles of the mean concentration. This figure clearly shows that the concentration noise was very small compared to the peak mean value. Moreover, it is evident that the mean concentration distribution could be fitted fairly well by Gaussian functions at all measurement planes, with the possible exception of the case at $x_{1} / L=5$, where the measured concentration peak slightly exceeded the Gaussian value, presumably due to persistence of injection effects.

The standard deviations $c^{\prime}$ of the concentration values at each pixel of all maps recorded on each plane were also calculated and fitted by Gaussian functions as

$$
\frac{c^{\prime}}{C_{S}}=A^{\prime} \exp \left[-\frac{\left(x_{2}-\mu_{2}^{\prime}\right)^{2}}{2 \sigma_{2}^{\prime 2}}-\frac{\left(x_{3}-\mu_{3}^{\prime}\right)^{2}}{2 \sigma_{3}^{\prime 2}}\right]
$$




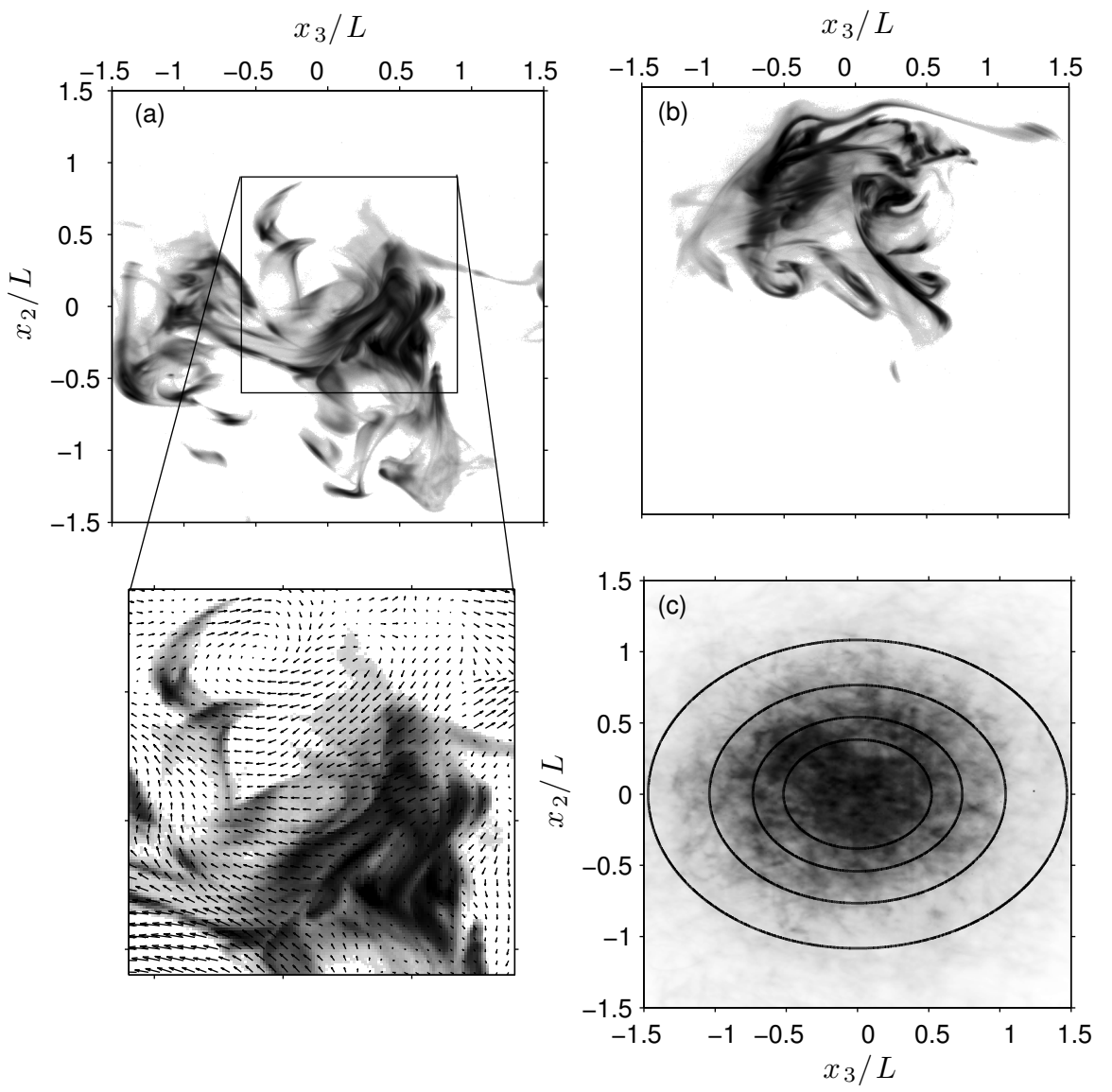

Figure 5: (a-b) Representative maps of the normalized instantaneous concentration $C /\left(C_{S} A\right)$ at $x_{1} / L=28$, with a blown-up view also showing superimposed an in-plane velocity vector map; (c) map of the normalized mean concentration $\bar{C} /\left(C_{S} A\right)$, also at $x_{1} / L=28$, with isocontours of the fitted 2D Gaussian function indicated by ellipses.
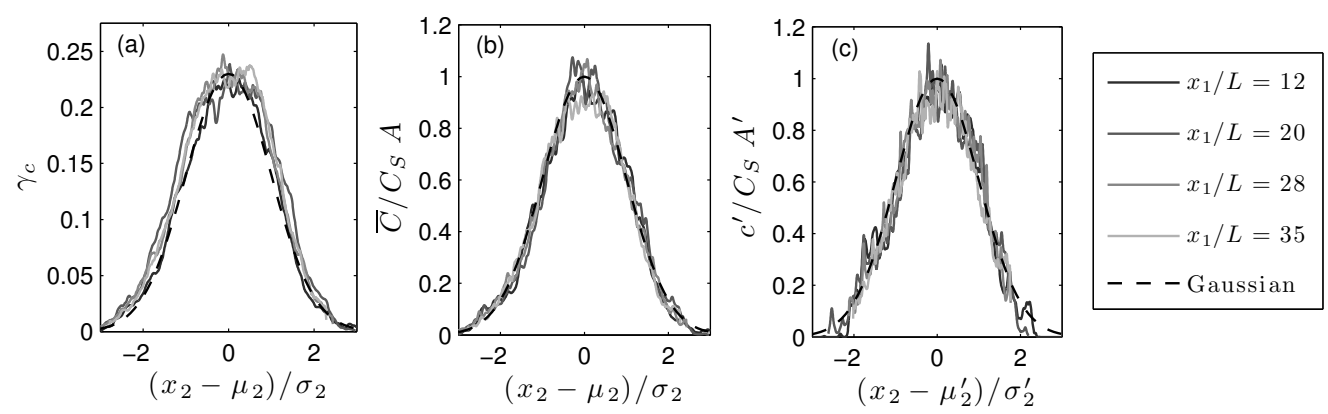

Figure 6: Transverse profiles of (a) the intermittency factor, (b) the normalized mean concentration, and (c) the normalized standard deviation of the concentration fluctuations. 

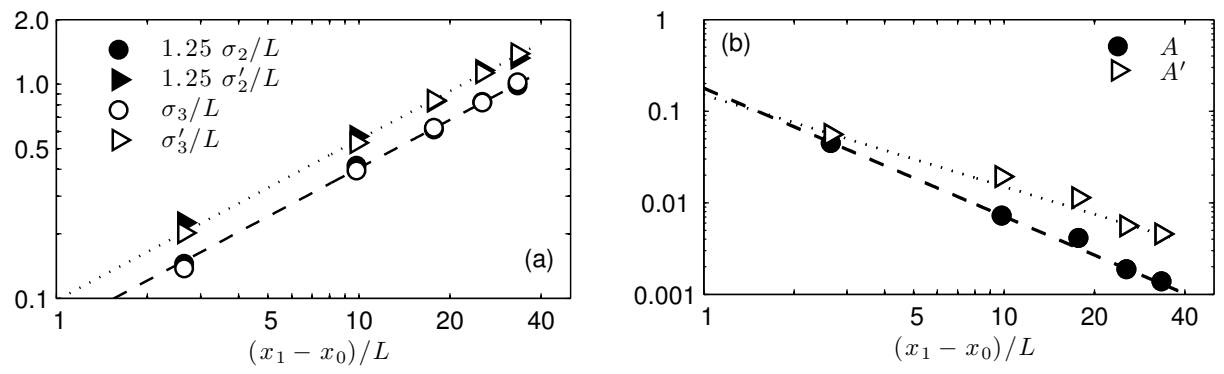

Figure 7: (a) Streamwise evolutions of the half-widths $\sigma_{2}$ and $\sigma_{3}$ of the mean concentration maps and the half-widths $\sigma_{2}^{\prime}$ and $\sigma_{3}^{\prime}$ of the corresponding standard deviation maps; all widths have been normalized by $L$; a dashed line indicates the power law $0.072\left[\left(x_{1}-x_{0}\right) / L\right]^{0.75}$ and a dotted line indicates the power law $0.098\left[\left(x_{1}-x_{0}\right) / L\right]^{0.75}$; (b) streamwise evolutions of $A$ and $A^{\prime}$, which are, respectively, the dimensionless amplitudes of the Gaussian fits to the mean concentration maps and the corresponding standard deviation maps; a dashed line indicates the fitted power law $A=$ $0.18\left[\left(x_{1}-x_{0}\right) / L\right]^{-1.4}$ and a dashed line indicates $A^{\prime}=0.15\left[\left(x_{1}-x_{0}\right) / L\right]^{-1.0} ;$ in all cases the virtual origin was $x_{0}=2.0 \mathrm{~L}$.

where the parameters $A^{\prime}, \mu_{2}^{\prime}, \mu_{3}^{\prime}, \sigma_{2}^{\prime}$ and $\sigma_{3}^{\prime}$ were determined as those that created the best fits to the measured $c^{\prime}$ at every pixel, using the same least-squares algorithm as for the mean concentration maps and starting with the same initial guesses. Transverse profiles of the normalized $c^{\prime}$ are presented in figure $6 \mathrm{c}$, which makes it evident that Gaussian fits were fairly good at all locations.

In the following, $\sigma_{2}$ and $\sigma_{3}$ will be referred to, respectively, as the transverse and spanwise half-widths of the mean concentration map. Similarly, $\sigma_{2}^{\prime}$ and $\sigma_{3}^{\prime}$ will be referred to, respectively, as the transverse and spanwise half-widths of the concentration fluctuation map (i.e., of $c^{\prime}$ ). The streamwise evolutions of the various plume half-widths are shown in figure $7 \mathrm{a}$. The half-widths of the fluctuation maps were greater than those of the corresponding mean maps. All half-widths grew monotonically downstream and could be fitted by power laws, with powers equal to 0.75 . The spanwise half-widths were consistently larger than the transverse ones, with $\sigma_{3} / \sigma_{2}$ and $\sigma_{3}^{\prime} / \sigma_{2}^{\prime} \approx 1.25$. This difference is attributed to the fact that $u_{3}^{\prime}>u_{2}^{\prime}$ in USF.

The fitted amplitudes $A$ and $A^{\prime}$ are plotted in figure 7b. Both amplitudes decreased as the plume spread, following the power laws $A=0.18\left[\left(x_{1}-x_{0}\right) / L\right]^{-1.4}$ and $A^{\prime}=$ $0.15\left[\left(x_{1}-x_{0}\right) / L\right]^{-1.0}$, with the same virtual origin of $x_{0}=2.0 L$. The decrease in the mean concentration indicates that the plume is being mixed with freestream fluid, whereas the decrease in the standard deviation indicates that the plume tends to become more homogeneous (Webster et al. 2003). The variation of the ratio $A^{\prime} / A$ depends on the evolution of the intermittency factor, as can be demonstrated by the following idealized analysis. Consider a binary signal with values 0 and 1 , as an idealized model of the concentration variation in the present plume. The probability distribution of such a signal, known as the Bernoulli distribution, has a mean equal to $\gamma_{c}$ and a standard deviation equal to $\sqrt{\gamma_{c}\left(1-\gamma_{c}\right)}$. For $\gamma_{c}<0.5$, the standard deviation of the binary random variable would always exceed its mean, in conformity with our observation in the plume. Furthermore, as the intermittency factor decreases, the mean of the binary random variable would decrease at a faster rate than its standard deviation, which is also consistent with the 
increasing ratio $A^{\prime} / A$ along the axis of the plume, where the peak $\gamma_{c}$ decreased from 0.4 to 0.23 between $x_{1} / L=5$ and 12 .

The estimated displacements of the transverse plume axis position $\mu_{2}$ and $\mu_{2}^{\prime}$ increased gradually to a few millimetres above the $x_{1}$ axis far downstream of the source. In view of the slight streamline displacement due to boundary layer growth on the water tunnel bottom, the uncertainty of alignment of the measurement planes at different downstream positions and the effect of finite population of samples used for calculating these values, it would be unwise to attach much significance to this observation. For this reason, we will refrain from claiming that a systematic effect of the mean shear is to displace the plume axis in the direction of the mean velocity gradient, even slightly so. It is noted that Tavoularis \& Corrsin (1981), Nakamura et al. (1986) and Karnik \& Tavoularis (1989) reported that their plume axes drifted towards the lower velocity region of their USF; all these displacements were also relatively small. The estimated displacements of the spanwise plume axis position $\mu_{3}$ and $\mu_{3}^{\prime}$ were also found to reach values of a few millimetres far downstream. In conformity with the previous discussion, we will also attribute this to uncertainties and not to a systematic asymmetry in the flow.

\subsection{Concentration-velocity correlations}

The covariances of the concentration and the velocity components were calculated following resampling of the SPIV measurements to the same grid as the PLIF ones. Interpolated values in the velocity field were determined by cubic spline interpolation of the values at neighbouring grid points, implemented using the scientific computation package MATLAB. As the spatial resolution of the velocity field was comparable to the Kolmogorov lengthscale of the flow, velocity changes between measurement points would be relatively small, which justifies interpolation between grid points; moreover, we found that maps of the concentration-velocity covariance were insensitive to the choice of interpolation scheme.

Because of the extremely strong concentration intermittency in the current plume, all correlation coefficients of the concentration had lower magnitudes compared with those obtained in flows with more homogeneous scalar fields. Following Wilson et al. (1985), we defined the mean conditional concentration $\overline{C_{P}}$ as the average of only non-zero values. This implies that the mean conditional concentration is related to the mean concentration as

$$
\overline{C_{P}}=\frac{1}{\gamma_{c}} \bar{C} .
$$

By extending this relationship to concentration fluctuations, we may estimate a conditional concentration-velocity correlation coefficient as

$$
\left[\overline{c u_{\alpha}}\right]_{c^{\prime} u_{\alpha}^{\prime}} \approx \frac{1}{\gamma_{c}} \frac{\overline{c u_{\alpha}}}{\overline{c^{\prime} u_{\alpha}^{\prime}}} .
$$

When comparing flows with vastly different scalar intermittencies, it seems more appropriate to compare conditional rather than conventional values. Transverse profiles of the correlation coefficients $\overline{c u_{\alpha}} / c^{\prime} u_{\alpha}^{\prime}$, corrected by the local intermittency factor $\gamma_{c}$, are presented in figure 8 . For $x_{1} / L>20$, the profiles were self-similar, and appeared to be essentially linear. Unlike conventional correlation coefficients, the conditional ones in the present plume are in good agreement with measurements in a much less intermittent thermal plume (Karnik \& Tavoularis 1989), which were corrected assuming that their plume had an intermittency factor of 1 on the plume centreline, approached zero at the plume edges, and also followed a Gaussian profile. In general, $\overline{c u_{1}} / c^{\prime} u_{1}^{\prime}$ and $\overline{c u_{2}} / c^{\prime} u_{2}^{\prime}$ had opposite signs, and reversed sign near the plume axis. Lastly, we note that while the 

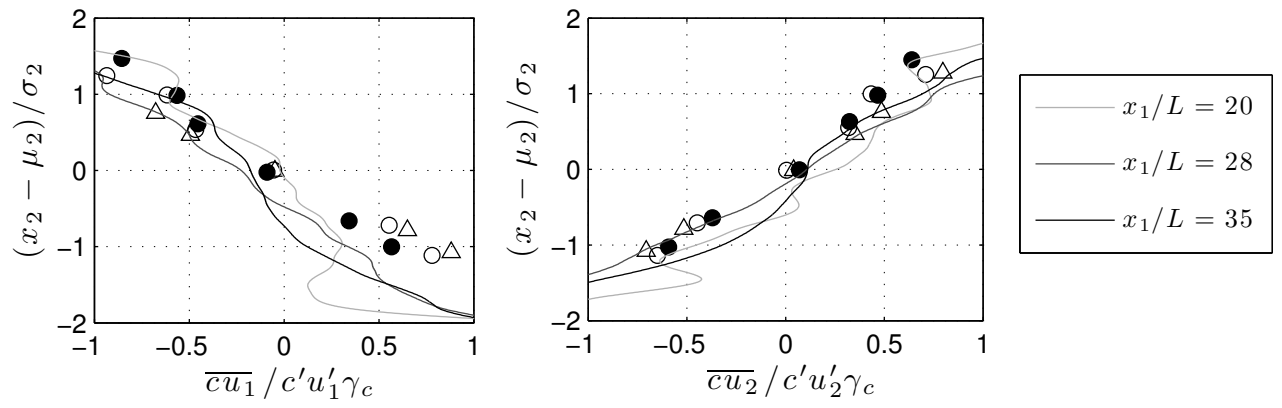

Figure 8: Transverse profiles of the velocity-concentration correlation coefficients corrected by the local intermittency factor $\gamma_{c}$, compared with corrected measurements from Karnik \& Tavoularis (1989) in a thermal plume at $x_{1} / L=24(\bullet), 48(\bigcirc), 84(\triangle)$.

profiles of $\overline{c u_{2}} / c^{\prime} u_{2}^{\prime}$ were nearly symmetric, the profiles of $\overline{c u_{1}} / c^{\prime} u_{1}^{\prime}$ all had slightly lower magnitudes in the lower half of the plume than in the upper half; this difference is associated with the presence of counter-gradient streamwise diffusion and will be discussed in detail in section 5.5 .

\subsection{Estimates of turbulent diffusivities}

In the present experiments, cross-sectional maps of all components of the turbulent mass flux vector $-\overline{c u_{i}}$ were obtained following resampling and interpolation of the SPIV measurements to the same grid as the PLIF ones. Cross-sectional maps of all components of the mean concentration gradient $\partial \bar{C} / \partial x_{i}$ were also independently determined by analytical differentiation of (4.2). This permitted the calculation of all nine components of the turbulent diffusivity tensor $D_{i j}$ as those values that resulted in the best fit between the left- and right-hand sides of (1.2) over each set of corresponding maps. The cross components $D_{13}, D_{23}, D_{31}$ and $D_{32}$ should vanish because of the symmetry of the Reynolds stress tensor and the scalar field about the $\left(x_{1}, x_{3}\right)$ plane. Moreover, preliminary calculations confirmed that the effects of these components were indeed negligible in the present flow. Consequently, these diffusivities were set to zero before calculating the other components.

Representative maps of the calculated mean scalar derivatives at $x_{1} / L=28$ are presented in figures 9a-c. Figures $9 \mathrm{~d}-\mathrm{f}$ show representative maps of scalar flux measurements and contours of the corresponding components of $D_{i j} \partial \bar{C} / \partial x_{j}$. Figures 9g-i show crosssections of the scalar flux maps, together with profiles of the appropriate individual terms of $D_{i j} \partial \bar{C} / \partial x_{j}$ and their sums, which demonstrate that (1.2) was consistent with all measurements.

The streamwise evolution of the estimated $D_{22}$ is plotted in figure 10a, whereas figures 10d-g show the evolutions of the ratios of the remaining non-zero diffusivities and $D_{22}$. It may be seen that there is considerable uncertainty in these results, much of which is attributed to the uncertainty of the estimated streamwise scalar derivative.

Secondary estimates of the turbulent diffusivities were obtained by assuming that gradient transport also applies to third-order concentration-velocity covariances, as (Karnik \& Tavoularis 1989)

$$
-\overline{c^{2} u_{i}}=D_{i j} \frac{\partial c^{\prime 2}}{\partial x_{j}}
$$

and estimating maps of $\partial c^{2} / \partial x_{j}$ by analytical differentiation of (4.3). These estimates, 

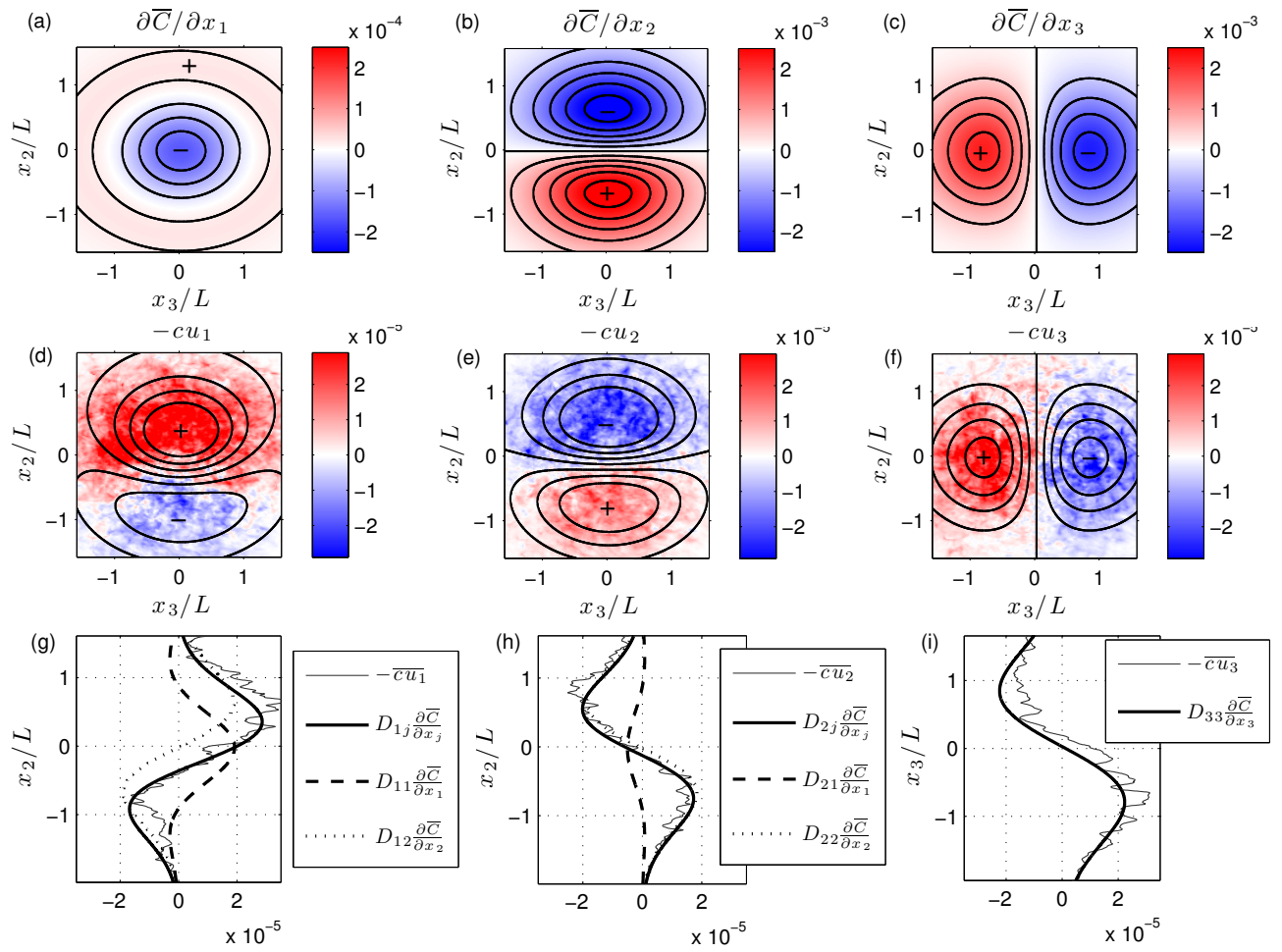

Figure 9: (a-c) Maps of the calculated mean concentration derivatives at $x_{1} / L=28$, normalized by $C_{S} / L$; (d-f) maps of $-\overline{c u_{i}}$ measurements at $x_{1} / L=28$, normalized by $C_{S} U_{C}$; black lines mark contours of the corresponding components of $D_{i j} \partial \bar{C} / \partial x_{j}$, also normalized by $C_{S} U_{C}$; the symbols + and - indicate regions with positive and negative values; (g-i) cross-sections of the $-\overline{c u_{i}}$ maps with profiles of the appropriate individual terms of $D_{i j} \partial \bar{C} / \partial x_{j}$ and their sums, all normalized by $C_{S} U_{C}$.

obtained as the best fits of maps of $-\overline{c^{2} u_{i}}$ to sets of maps of $\partial c^{\prime 2} / \partial x_{j}$, have also been plotted in figure 10. It can be seen that the two estimates of each diffusivity are generally close to each other, although it may be noted that the latter estimates have higher uncertainty than the former ones.

The turbulent Schmidt number $S c_{T} \equiv \nu_{T} / D_{22}$ decreased along the plume (see figure 10c) and approached an asymptote of approximately 1.3 for $x_{1} / L \geqslant 28$. This value is somewhat larger than the value of 1.1 measured by Tavoularis \& Corrsin (1981) and the conventional value near unity (Pope 2000).

The apparent diffusivities $K_{2}$ and $K_{3}$ were calculated by differentiating the power laws fitted to the corresponding half-widths, according to (2.7). This operation also provided power laws for $K_{2}$ and $K_{3}$, as, for example, $K_{2} /\left(U_{C} L\right)=0.0025\left[\left(x_{1}-x_{0}\right) / L\right]^{0.5}$, where, as previously, the virtual origin was set as $x_{0}=2.0 \mathrm{~L}$. The apparent diffusivity ratio was $K_{3} / K_{2}=\left(\sigma_{3} / \sigma_{2}\right)^{2}=1.56$. The evolution of the transverse apparent diffusivity $K_{2} /\left(U_{C} L\right)$, plotted in figure 10a, is fairly consistent with the measured values of $D_{22} /\left(U_{C} L\right)$, although it tends to slightly overshoot the measurements in the far field. Similarly, the apparent Schmidt number $\nu_{T} / K_{2}$, plotted in figure $10 \mathrm{c}$, is consistent with the values determined using $D_{22}$. Furthermore, the ratio $K_{3} / K_{2}$ is close to measurements of $D_{33} / D_{22}$, as shown 

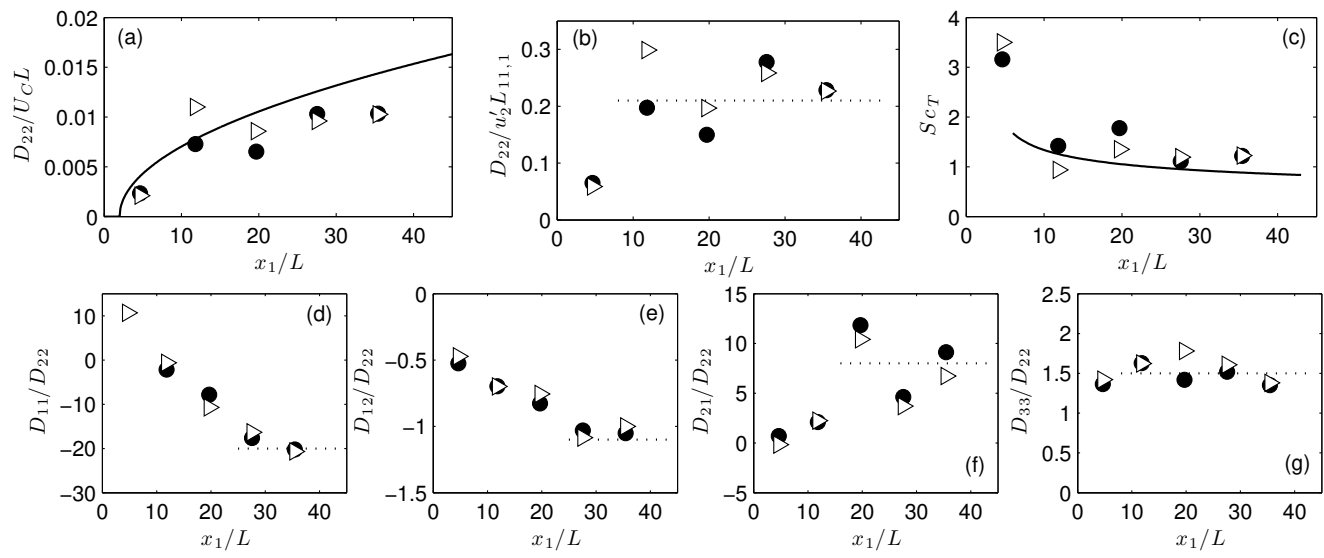

Figure 10: (a) The estimated turbulent diffusivity $D_{22} / U_{C} L$ (symbols) and the apparent diffusivity $K_{2} / U_{C} L$ (solid line); (b) $D_{22} / u_{2}^{\prime} L_{11,1}$ (symbols) and its estimated asymptote (dotted line), with $L_{11,1} \approx L_{22,2} / 0.55$; (c) estimates of the turbulent Schmidt number $S c_{T}=\nu_{T} / D_{22}$ and the apparent Schmidt number $\nu_{T} / K_{2}$ (solid line); (d-g) ratios of the turbulent diffusivities (symbols) and their estimated asymptotes (dotted lines); $\bullet$ and $\triangleright$ indicate diffusivities estimated from (1.2) and (4.6), respectively.

in figure 10g. These observations support the use of the apparent diffusivities $K_{2}$ and $K_{3}$ as surrogates for the turbulent diffusivities $D_{22}$ and $D_{33}$.

Taylor predicted that the turbulent diffusivity would grow to a state in which it would depend only on Lagrangian turbulence properties when $\mathcal{T} \ll t$. For a continuously released plume in steady flow, this state would be approached at a downstream distance that would be comparable to the streamwise Lagrangian integral lengthscale $\mathcal{L}_{11}$. Although we have no measurements of this lengthscale in the present flow, we can estimate it for USF as $\mathcal{L}_{11}=2 \mathcal{L}_{22}$ (Tavoularis \& Corrsin 1985), where $\mathcal{L}_{22}$ is the transverse lengthscale, which in turn can be estimated as $\mathcal{L}_{22} \approx 1.3 L_{22,2} / u_{2}^{\prime}$ (Karnik \& Tavoularis 1990). Using these approximations, we obtained $\mathcal{L}_{11} \approx 45 L$, which is slightly higher than the current range of measurements. Nevertheless our measurements of $D_{22} / u_{2}^{\prime} L_{11,1}$ appear to approach a constant value, in conformity with Taylor's conjecture. The value of this constant was approximately 0.21 (see figure $10 \mathrm{~b}$ ), which is approximately twice the value reported by Karnik \& Tavoularis (1989).

When normalized by $D_{22}$, most components of the turbulent diffusivity tensor appeared to approach asymptotes far downstream of the source (figures $10 \mathrm{~d}-\mathrm{g}$ ); the ratio $D_{11} / D_{22}$ seems to be an exception, as it keeps becoming more negative with increasing downstream distance. The mean values of the ratios for $x_{1} / L \geqslant 28$ were

$$
\frac{D_{i j}}{D_{22}} \approx\left[\begin{array}{rcl}
-20 & -1.1 & 0 \\
8 & 1.0 & 0 \\
0 & 0 & 1.5
\end{array}\right] .
$$

The presence of non-zero cross-diffusivities and the fact that $D_{11}, D_{22}$ and $D_{33}$ had different values are consequences of the strong anisotropy of the turbulence in USF. The cross-diffusivities introduce effects of more than one mean scalar derivatives to the each component of the scalar flux vector, a result that is also attributable to shear-generated anisotropy. One may make the following observations concerning individual scalar fluxes.

The spanwise flux $\left(-\overline{c u_{3}}\right)$ : This property was proportional to the spanwise mean 
scalar derivative. Consistent with the fact that $\overline{u_{1} u_{3}}=\overline{u_{2} u_{3}}=0$ in USF, $D_{31}=D_{32}=0$, so $-\overline{c u_{3}}$ had no contributions from the transverse and streamwise mean scalar derivatives. The ratio $D_{33} / D_{22}$ was approximately 1.5 , which is sufficiently close to the values of the ratios $u_{3}^{\prime 2} \mathcal{T}_{33} / u_{2}^{\prime 2} \mathcal{T}_{22}$ and $K_{3} / K_{2}$ and the result reported by Tavoularis \& Corrsin (1985).

The transverse flux $\left(-\overline{c u_{2}}\right)$ : The transverse flux had contributions from both the transverse and the streamwise mean scalar derivatives, although the contribution of the transverse derivative dominated, as illustrated in figure $9 \mathrm{~h}$. The value of $D_{21}$ appeared to be positive, albeit within considerable uncertainty, as the term $D_{21} \partial \bar{C} / \partial x_{1}$ was much smaller than $D_{22} \partial \bar{C} / \partial x_{2}$.

The streamwise flux $\left(-\overline{c u_{1}}\right)$ : The streamwise flux had contributions from both the transverse and the streamwise mean scalar derivatives. As illustrated in figure $9 \mathrm{~g}$, these contributions had, respectively, antisymmetric and symmetric profiles. The antisymmetric contribution corresponded to $D_{12} / D_{22} \approx-1.1$, which is consistent in sign but lower in magnitude than the values -2.2 and -2.0 , reported, respectively, by Tavoularis \& Corrsin (1981) and Karnik \& Tavoularis (1989). The negative sign of $D_{12}$ is a consequence of the fact that, in USF, the streamwise velocity fluctuations $u_{1}$ are negatively correlated to the transverse fluctuations $u_{2}$ (Tavoularis \& Corrsin 1981). Because of the near symmetry of the transverse mean scalar derivative $\partial \bar{C} / \partial x_{2}$, its contribution to the streamwise flux was negligible along the plume axis; therefore, the non-zero values of $-c u_{1}$ may be attributed to the streamwise mean scalar derivative. Far downstream of the source, $-c u_{1}>0$ and $\partial \bar{C} / \partial x_{1}<0$ along the plume axis, hence $D_{11}$ was negative (i.e., counter-gradient). Although $\left|\partial \bar{C} / \partial x_{1}\right| \ll\left|\partial \bar{C} / \partial x_{2}\right|$, the term $D_{11} \partial \bar{C} / \partial x_{1}$ in the core of the plume was comparable in magnitude to the peak values of $D_{12} \partial \bar{C} / \partial x_{2}$, with the result that $D_{11}$ was an order of magnitude larger than $D_{12}$. Because $\left|\partial \bar{C} / \partial x_{1}\right|$ was small, the magnitude of $D_{11}$ is subject to more uncertainty than the other terms, which is why we only report the value of $D_{11}$ to one significant digit; however, the fact that estimates from both (1.2) and (4.6) are consistent attests to the accuracy of the measured diffusivities. For additional discussion concerning the complex shape of $-\overline{c u_{1}}$ and the negative value of $D_{11}$, please see section 5.5.

\section{Analysis of the results and discussion}

\subsection{The plume width}

It is noted that our definition of plume width differs from those used by previous authors; Webster et al. (2003) defined the plume half-width as twice the standard deviation of the mean concentration profile, whereas Karnik \& Tavoularis (1989) defined it as half the distance between locations with mean concentrations equal to half the peak value. Therefore, according to the present definition, the transverse half-widths of the plumes examined by Webster et al. and Karnik \& Tavoularis would, respectively, be equal to 0.5 and 0.85 times the values reported by the corresponding authors.

The evolution of the half-widths could be fitted by power laws with an exponent of 0.75 . This value is equal to the value reported by Webster et al., within the range of 0.55 to 0.76 reported by Lepore \& Mydlarski (2011), and comparable to the value 0.83 fitted by us to the data of Karnik \& Tavoularis.

When comparing measurements in the present plume to those from the literature, it is also important to consider the state of growth of each plume with respect to the local size of the turbulent eddies which convect and diffuse the scalar field. The lengthscale ratio $\sigma_{2} / L_{22,2}$ may be chosen as a measure of relative plume growth. The streamwise evolutions of this ratio in the present measurements and in the previous study by Karnik \& 


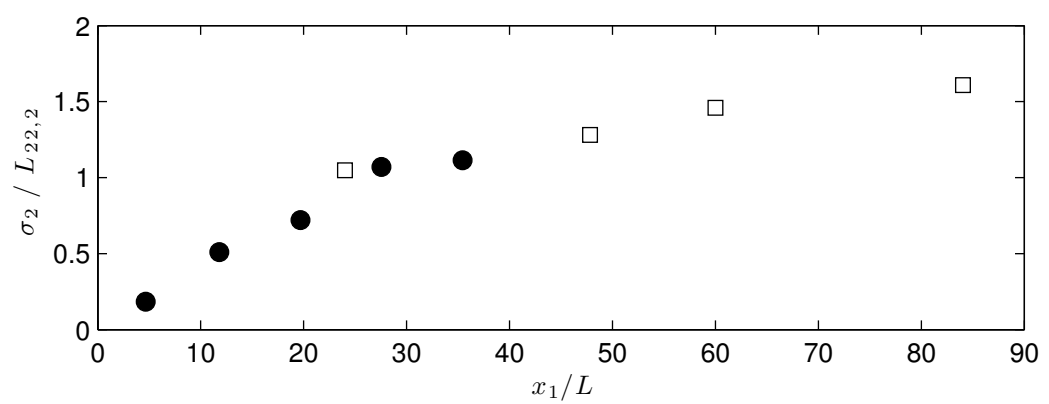

Figure 11: Downstream development of the ratio of the plume halfwidth $\sigma_{2}$ and the transverse turbulent integral lengthscale $L_{22,2} ; \bullet$, present measurements; $\square$, Karnik \& Tavoularis (1989), with $L_{22,2} \approx 0.55 L_{11,1}$.

Tavoularis (1989) is presented in figure 11. As figure 11 illustrates, our facility permitted the present plume width to grow to values that were comparable to the transverse integral lengthscale and so the entire plume may be characterised as slender. This was not the case with the plume studied by Karnik \& Tavoularis (1989), which grew to measurably larger relative thicknesses.

\subsection{Scalar fluctuations}

All present profiles of $c^{\prime}$ (see figure $7 \mathrm{~b}$ ) were single-peaked, unlike some of the profiles presented by Warhaft (1984), Karnik \& Tavoularis (1989), Rahman \& Webster (2005), and Lepore \& Mydlarski (2011), which were double-peaked. Karnik \& Tavoularis attributed the presence of double peaks in the near-source profiles to the particular shape of the thermal wake of the heated ribbon used, a feature that is absent in the present experiments. The development of double peaks far downstream of the source in the previous studies was attributed to the fact that the peak production of scalar variance occurs at the inflection points of the mean concentration field, which are located on either side of the plume centreline. However, for gradient transport to be effective in producing local fluctuation peaks, the plume width must be sufficiently large with respect to the integral lengthscale of the flow. The logic behind this explanation is that, when the plume is large enough for the local scalar transport to be dominated by eddy motions that are mostly confined to the same side of the plume, scalar fluctuations would follow gradient transport and so they would peak at the two inflection points of the mean concentration gradient; on the other hand, when the plume half width is small by comparison to the turbulent eddy mean free path, local transport would be dominated by eddy motions that sweep across much of or the entire plume and thus smoothen off-axis peaks of concentration fluctuations. This argument, however, is not sufficient to explain entirely the lack of double peaks in the present plume, whose half width grew to approximately the size of the transverse integral lengthscale. An explanation will be given in the following paragraph.

The present plume differs from the one studied by Warhaft (1984), Karnik \& Tavoularis (1989), and Lepore \& Mydlarski (2011) in an important aspect: the former originated from essentially a point source, whereas the latter originated from a line-source and was two-dimensional on the mean. In a line-source plume, strong scalar fluctuations would be introduced mainly by transverse motions (i.e., in a direction parallel to the mean scalar gradient), whereas in a point-source plume, motions in all directions on a transverse plane may introduce strong scalar fluctuations. Therefore, if both plumes had the same 

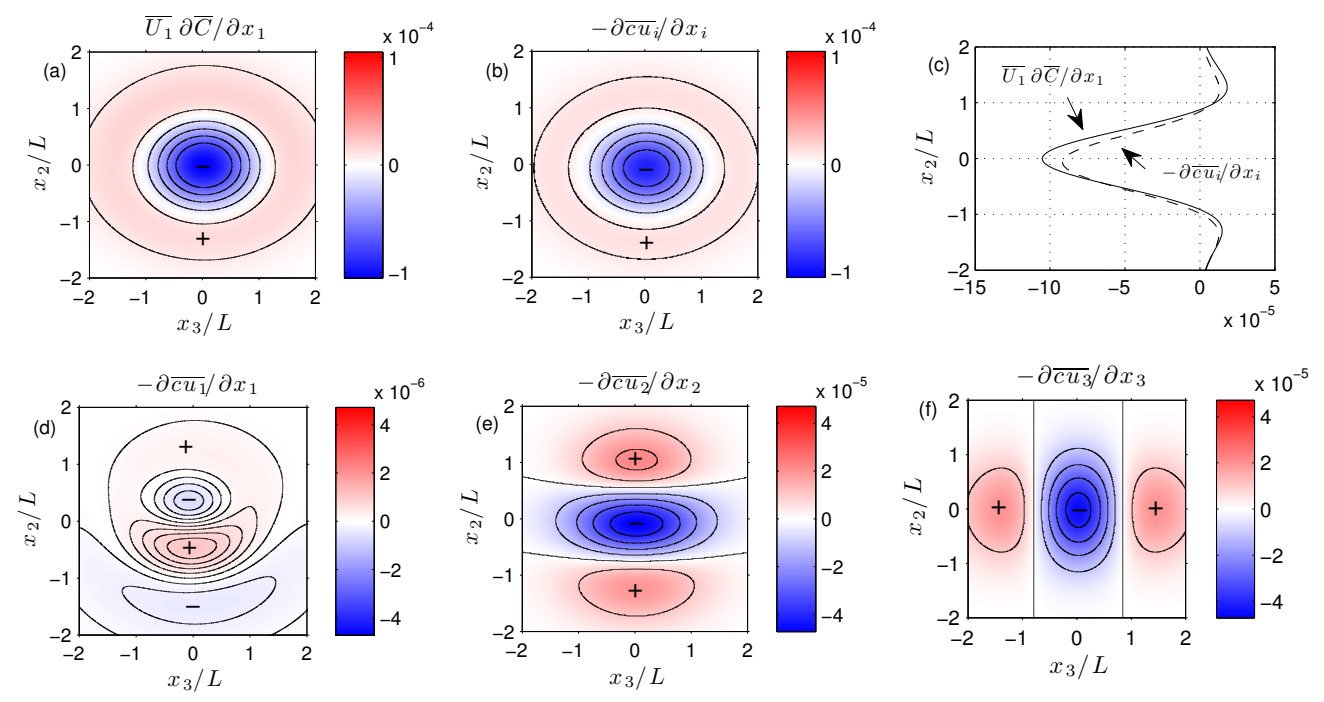

Figure 12: (a) Maps of the advective flux; (b) maps of the net diffusive flux in (1.1); (c) transverse profiles of the advective and net diffusive fluxes at $x_{3}=0 ;(\mathrm{d}-\mathrm{f})$ maps of the individual diffusive flux terms; all results were at $x_{1} / L=28$; all fluxes were normalized by $C_{S} U_{C} / L$ and the symbols + and - indicate map regions with positive and negative values, respectively.

ratio of $\sigma_{2} / L_{22,2}$, the 2-D plume would have a stronger tendency to be double-peaked. One may then speculate that, for a double-peak $c^{\prime}$ to be manifested in a point-source plume, $\sigma_{2} / L_{22,2}$ would have to be much larger than its double-peaking threshold for a line-source plume. The present plume was clearly far from meeting this condition, which explains the observed single-peak pattern.

\subsection{Estimates of advection and diffusion}

In this section, we investigate the terms in the Reynolds-averaged advection-diffusion equation (1.1). The advection term in (1.1), which in USF is simplified to $\overline{U_{1}} \partial \bar{C} / \partial x_{1}$, was calculated by multiplying the local mean velocity by the mean streamwise concentration derivative, estimated by analytical differentiation of (4.2). The molecular diffusion term was negligible, as the molecular diffusivity was several orders of magnitude smaller than the main turbulent diffusivity. To estimate the three turbulent diffusion terms, we first created smooth maps of $-\overline{c u_{1}},-\overline{c u_{2}}$ and $-\overline{c u_{3}}$ using (4.2) and (1.2) and the estimated turbulent diffusivities. The streamwise diffusive flux term $-\partial \overline{c u_{1}} / \partial x_{1}$ was then determined applying first-order central differencing to data from five measurement planes. The inplane diffusive flux terms $-\partial \overline{c u_{2}} / \partial x_{2}$ and $-\partial \overline{c u_{3}} / \partial x_{3}$ were determined using second-order central differencing. Maps of the advection term, the three diffusive flux terms, and the net (total) diffusion term are presented in Figure 12.

The estimated maps of the advection and net diffusion terms, which should ideally balance each other, are in fairly good agreement; this attests to the accuracy of our results. The net diffusion term appears to have somewhat smaller magnitude than the advection term (see figure 12c); this difference may be largely attributed to the uncertainty of the streamwise derivative of the mean concentration. The magnitude of this discrepancy was comparable to the one in a turbulent jet (Fukushima et al. 2000), which was also attributed to insufficient spatial resolution. 
Tavoularis \& Corrsin (1985)

$$
\begin{aligned}
& D_{11}=\overline{u_{1}^{2}} \mathcal{T}_{11}+\frac{d \overline{U_{1}}}{d x_{2}} \overline{u_{1} u_{2}} \mathcal{T}_{12}^{2} \\
& D_{12}=\overline{u_{1} u_{2}} \mathcal{T}_{12} \\
& D_{21}=\overline{u_{1} u_{2}} \mathcal{T}_{21}+\frac{d \overline{U_{1}}}{d x_{2}} \overline{u_{2}^{2}} \mathcal{T}_{22}^{2} \\
& D_{22}=\overline{u_{2}^{2}} \mathcal{T}_{22} \\
& D_{33}=\overline{u_{3}^{2}} \mathcal{T}_{33}
\end{aligned}
$$

Rogers et al. (1989)

$$
\begin{aligned}
& D_{11}=\overline{u_{1}^{2}} \mathcal{T}-\frac{d \overline{U_{1}}}{d x_{2}} \overline{u_{1} u_{2}} \mathcal{T}^{2} \\
& D_{12}=\overline{u_{1} u_{2}} \mathcal{T}-\frac{d \overline{U_{1}}}{d x_{2}} \overline{u_{2}^{2}} \mathcal{T}^{2} \\
& D_{21}=\overline{u_{1} u_{2}} \mathcal{T} \\
& D_{22}=\overline{u_{2}^{2}} \mathcal{T} \\
& D_{33}=\overline{u_{3}^{2}} \mathcal{T}
\end{aligned}
$$

Younis et al. (2005)

$$
\begin{aligned}
& D_{11}=\overline{u_{1}^{2}}\left(C_{2} \frac{k}{\epsilon}\right)+\frac{d \overline{U_{1}}}{d x_{2}} \overline{u_{1} u_{2}}\left(2 C_{4} \frac{k^{2}}{\epsilon^{2}}\right)+C_{1} \frac{k^{2}}{\epsilon} \\
& D_{12}=\overline{u_{1} u_{2}}\left(C_{2} \frac{k}{\epsilon}\right)+\frac{d \overline{U_{1}}}{d x_{2}} \overline{u_{2}^{2}}\left(C_{4} \frac{k^{2}}{\epsilon^{2}}\right)+C_{3} \frac{k^{3}}{\epsilon^{2}} \frac{d \overline{U_{1}}}{d x_{2}} \overline{u_{2}^{2}} \\
& D_{21}=\overline{u_{1} u_{2}}\left(C_{2} \frac{k}{\epsilon}\right)+\frac{d \overline{U_{1}}}{d x_{2}} \overline{u_{2}^{2}}\left(C_{4} \frac{k^{2}}{\epsilon^{2}}\right) \\
& D_{22}=\overline{u_{2}^{2}}\left(C_{2} \frac{k}{\epsilon}\right)+C_{1} \frac{k^{2}}{\epsilon} \\
& D_{33}=\overline{u_{3}^{2}}\left(C_{2} \frac{k}{\epsilon}\right)+C_{1} \frac{k^{2}}{\epsilon}
\end{aligned}
$$

Table 3: Theoretical models of the turbulent diffusivities.

TC RMR YSC present

$\begin{array}{lrrrc}D_{22} / D_{22 m} & 3.2 & 2.3 & 3.8 & 1 \\ D_{11} / D_{22} & -10.0 & 2.8 & 3.4 & -20 \\ D_{33} / D_{22} & 1.6 & 1.6 & 1.9 & 1.5 \\ D_{12} / D_{22} & -2.0 & -1.9 & -1.8 & -1.1 \\ D_{21} / D_{22} & 0.0 & -0.5 & -1.5 & 8\end{array}$

Table 4: Theoretical estimates of the turbulent diffusivity ratios; $D_{22 m}$ indicates the measured $D_{22}$.

Comparing the maps of the three diffusive flux terms, it is evident that the spanwise term was essentially symmetric; the transverse term was slightly asymmetric, as a result of the non-zero value of $D_{21}$; the streamwise term was strongly asymmetric, in conformity with the complex shape of $-\overline{c u_{1}}$. The transverse and spanwise diffusive fluxes were nearly equal around the plume axis, but each became dominant off-axis, in regions in which the corresponding mean concentration derivative was dominant. The streamwise diffusive flux was consistently an order of magnitude smaller than the other two and made a very small contribution (less than $5 \%$ ) to the net diffusion. This provides justification for disregarding streamwise diffusion in simplified models, even though the streamwise diffusivity $D_{11}$ is much larger than the two other normal diffusivities.

\subsection{Comparison of diffusivities to theoretical estimates}

Analytical models of the turbulent diffusivity tensor relevant to USF have been developed by Tavoularis \& Corrsin (1985), Rogers et al. (1989), and Younis et al. (2005), to be referred to as TC, RMR and YSC, respectively. The corresponding expressions are summarized in table 3, whereas ratios of the predicted diffusivity values are compared to the present results in table 4 . All models assumed that $D_{31}=D_{13}=D_{32}=D_{23}=0$ by symmetry of the Reynolds stress tensor about the $\left(x_{1}, x_{3}\right)$ plane. 
The TC model contains the Lagrangian integral timescales $\mathcal{T}_{11}$ etc.. We have no measurements of these timescales in the present flow, but we used the estimates $\mathcal{T}_{22} \approx$ $1.3 L_{22,2} / u_{2}^{\prime}$ (Karnik \& Tavoularis 1990) and $\mathcal{T}_{12}=\mathcal{T}_{21}=4 \mathcal{T}_{22}, \mathcal{T}_{11}=2 \mathcal{T}_{22}$, and $\mathcal{T}_{33}=\mathcal{T}_{22}$ (TC), all applicable to USF. The RMR model is very similar to the TC model, however, the former contains a constant timescale $\mathcal{T}=2 k /\left(C_{D} \epsilon\right)$, in which $C_{D}=12.6$ for the present conditions $\left(C_{D}\right.$ is specified by RMR as a function mainly of a Reynolds number and, weakly, of the Prandtl number). The YSC model is more complex than either of the two other models, expressing the diffusivities in terms of relationships that contain four adjustable coefficients, for which YSC recommend values.

All models predicted values of $D_{22}$ which were more than double the measured value; moreover, they all predicted values of the ratio $D_{33} / D_{22}$ that were close to the experimental one. The TC prediction of $D_{11} / D_{22}$ agreed in sign with the measured ratio although it was smaller in magnitude, whereas the two other models missed this ratio not only in magnitude, but in sign as well. All models predicted $D_{12} / D_{22}$ that had the same sign as the measured value but were more than twice as large; in this respect, the models are in fair agreement with the measurements of Tavoularis \& Corrsin (1981) and Karnik \& Tavoularis (1989) who found $D_{12} / D_{22}$ was -2.2 and -2.0 , respectively. The RMR and YSC models predicted a negative value of $D_{21} / D_{22}$, whereas the TC model predicted a value of zero; in this respect, all models differ from the present measurements of $D_{21} / D_{22}$, which tended to be positive.

Overall, all models had comparable performances, with the notable exception that the TC model was the only one to predict correctly the sign of $D_{11}$. None of the models predicted accurately the magnitudes of the diffusivities, but predictions and measurements were of the same order of magnitude. It is noted that all models were developed and calibrated for air flows, in which the Prandtl/Schmidt numbers were of order one, whereas the scalar field in the present experiments had a very high Schmidt number.

\subsection{On the streamwise turbulent mass flux and the streamwise turbulent diffusivities}

The map of $-\overline{c u_{1}}$ (figure $9 \mathrm{~d}$ ) appears to be oddly complex and invites some in-depth physical scrutiny. For simplicity, let us consider the transverse profile of $-\overline{c u_{1}}$, shown in figure 9g. We shall explain its shape by the following qualitative discussion, which makes use of gradient-transport-type arguments, but also takes into consideration the turbulence structure of USF.

If this profile were entirely the result of transverse gradient transport, it would have been antisymmetric about the plume axis. In the lower half of the plume, where $\partial \bar{C} / \partial x_{2}>$ $0, c$ and $u_{2}$ would tend to be negatively correlated; however, in this USF, $u_{2}$ and $u_{1}$ are also negatively correlated, which implies that $c$ and $u_{1}$ would be positively correlated. A corresponding argument can be made for the upper half of the plume to show that $c$ and $u_{1}$ would be negatively correlated in this region. Using a similar argument, Tavoularis \& Corrsin (1981) explained the observed sign of $\overline{c u_{1}}$ in USF with a uniform mean scalar gradient. This argument explains why $D_{12}$ in shear flows would have the same sign as $\overline{u_{1} u_{2}}$.

The previous argument is not sufficient to explain the variation of $-\overline{c u_{1}}$, because its profile is obviously not antisymmetric. Nevertheless, $-\overline{c u_{1}}$ can be represented quite well by the sum of an antisymmetric part and a symmetric part. We will argue that the symmetric part may be attributed to the streamwise variation of the mean concentration and the plume growth. The supporting arguments can be best presented by considering the region near the plume axis, where the symmetric part is strongest and the antisymmetric part is weak. In this region, $\partial \bar{C} / \partial x_{1}<0$ and conventional gradient transport with a positive diffusivity $D_{11}$ would imply that $-\overline{c u_{1}}<0$ as well. This is not the case, however, because 
the symmetric part of $-\overline{c u_{1}}$ is strongly positive around the plume axis, thus necessitating the use of a negative $D_{11}$. This amounts to counter-gradient transport and requires further examination.

It was mentioned previously that the present plume half-width was comparable to the transverse turbulent lengthscale $L_{22,2}$, which means that the plume was subject to strong meandering so that fluid parcels from the edges of and outside the plume would often cross its axis. The value of $-\overline{c u_{1}}$ would be dominated by the contributions of events having simultaneously large fluctuations of $u_{1}$ and large fluctuations of $c$. Large negative fluctuations of $c$ would occur if an eddy originating at the plume edge or beyond approached the plume axis, as such an eddy would transport a concentration that would be much lower than the mean near the axis. For an eddy from the edges of the plume to penetrate to the axis, it must have a strong velocity $u_{2}$. In USF, such eddies would typically also have strong $u_{1}$ with a sign opposite to that of $u_{2}$. Eddies from the upper edge would have $u_{2}<0$ and $u_{1}>0$, i.e., they would originate upstream of the measurement plane. On the contrary, eddies from the lower edge would have $u_{2}>0$ and $u_{1}<0$, i.e., they would originate downstream of the measurement plane. So, eddies with large $c<0$ would sometimes be associated with $u_{1}>0$ and other times with $u_{1}<0$. How would then a net positive $-\overline{\mathrm{cu}}$ be produced near the axis?

We will now demonstrate that the positive $-\overline{c u_{1}}$ near the plume axis is the result of plume growth in size with streamwise distance from the origin, namely the result of transverse turbulent diffusion itself. In the plume core, $\partial \bar{C} / \partial x_{1}<0$, because the peak mean concentration decays downstream; on the contrary, $\partial \bar{C} / \partial x_{1}>0$ near the edges, because the plume spreads outwards. Consequently, upstream eddies crossing the measurement plane would transport negative $c$ with a magnitude that is larger than that of downstream eddies, which also transport negative $c$; thus, the net effect of mixing would be $-\overline{c u_{1}}>0$. This explains why $D_{11}$ was negative and transport was countergradient with respect to the gradient around the plume axis. This apparent paradox may, however, be resolved by a change of perspective: mass was actually flowing along the gradient, if one considers the gradient where it matters for $-\overline{c u_{1}}$, namely at the plume edges.

In the previous discussion, we invoked the fact that $u_{1}$ and $u_{2}$ are strongly correlated. In USF, this means that strong downward-bound eddies typically come from upstream and strong upward-bound eddies come from downstream, namely that the two types of eddies transport fluid from opposite edges of the plume. Albeit, because the plume is symmetric, it makes no difference to the value of $c$ whether it comes from the upper edge or the lower one. Consequently, counter-gradient streamwise transport does not require the net Reynolds stress to be non-zero, but only that eddies with strong fluctuations in $u_{1}$ also have strong $u_{2}$, which allows them to originate outside of the plume. In other words, mean shear is not necessary for counter-gradient transport to arise, but it helps, as it organizes the motion and generates strong coherent structures, which in the case of USF are horseshoe-shaped vortices (Vanderwel \& Tavoularis 2011).

We have so far focused on diffusion in the transverse direction, but there is also spanwise diffusion. In fact, Vanderwel \& Tavoularis (2011) demonstrated that coherent structures in USF are not always aligned with the transverse direction, but appear over a wide range of orientations. In the case of the present plume, strong eddies with significant spanwise velocity would still transport low-concentration fluid from the plume edges and beyond, and so they would contribute to counter-gradient transport. On the other hand, spanwise motions in two-dimensional plumes, such as those generated by a heated wire, would not encounter edges and so the range of motions that would contribute to counter-gradient transport would be severely restricted. This may explain why the transverse profiles of 
$-\overline{c u_{1}}$ measured by Karnik \& Tavoularis (1987) were essentially antisymmetric and free of streamwise diffusion effects.

The presently used scalar has a Schmidt number which is more than three orders of magnitude larger than the Prandtl number of air in heated-flow experiments, so it seems worthwhile to question whether this difference would play a significant role in countergradient transport. Molecular diffusion would reduce the contrast of concentration maps and lower concentration peaks, and so it would tend to reduce differences between concentration values in the core and the edges of the plume. Nevertheless, such effects would be present in both conventional and counter-gradient diffusion. In the absence of evidence for the opposite, one may speculate that counter-gradient diffusion is not subjected to strong Schmidt/Prandtl number effects.

To speculate whether a negative $D_{11}$ would also appear in a plume in another flow configuration, one would need to consider whether the plume width would satisfy the following conditions: first it should be sufficiently narrow in all directions by comparison to the lengthscale of the energy containing eddies and second it should grow sufficiently fast downstream. For example, in the case of a turbulent jet transporting a scalar in its entirety, the first condition would not be satisfied and so jets with $-\overline{c u_{1}}>0$ have not been reported (Fukushima et al. 2000; Webster et al. 2001). We have reviewed the available literature in search of previous references to counter-gradient streamwise diffusion, but could not find any. There are, however, several references dealing with counter-gradient diffusion in the transverse direction, with which the mean scalar gradient was nearly aligned. Such phenomena have been observed in atmospheric flows (Deardorff 1966; van Dop \& Verver 2001; de Roode et al. 2004) as well as in the laboratory (Sreenivasan et al. 1982; Veeravalli \& Warhaft 1990; Paranthoën et al. 2004) and in most cases the discrepancy has been attributed to inhomogeneity.

\section{Summary}

Simultaneous concentration and velocity maps in the plume of a continuous point source in uniformly sheared turbulence have been measured. Gradient transport analysis with a turbulent diffusivity tensor described well the relationship between the measured turbulent mass flux vector and the mean concentration gradient. For the first time, all non-vanishing components of the turbulent diffusivity tensor were determined simultaneously from experimental results. The apparent diffusivities followed the same trends as the corresponding normal diffusivities on a transverse plane. Sufficiently downstream of the source, the various components of the turbulent diffusivity tensor grew at the same rates with distance from the source. Counter-gradient streamwise diffusion was reported for the first time and was attributed to the meandering and streamwise spread of the plume. Three previous theoretical models of turbulent diffusion in shear flows had some qualitative agreement with the present results, especially the model of Tavoularis \& Corrsin (1985), which also predicted counter-gradient streamwise diffusion.

Financial support by the Natural Sciences and Engineering Research Council of Canada (NSERC) is gratefully acknowledged.

\section{Appendix A. Determining the optimal flow rate}

The optimal flow rate through the injector was determined by comparing the velocity maps for different injection flow rates in a plane normal to the flow at a distance of $x_{1} / L=5$ from the injector tip; this distance is equal to approximately 70 inner tube 


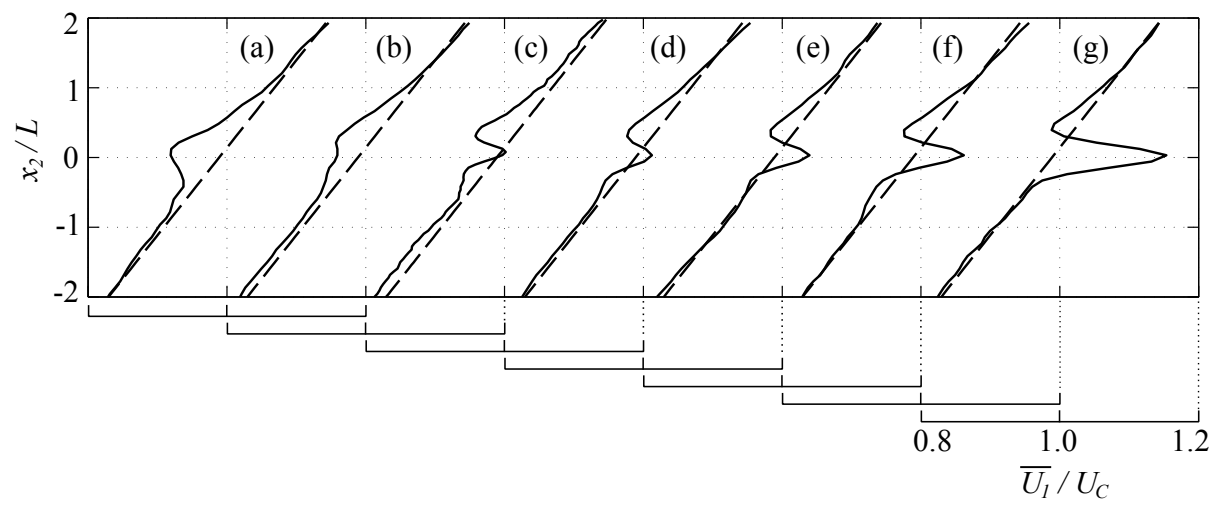

Figure 13: Solid lines are transverse profiles of the mean streamwise velocity at $x_{1} / L=5$ and $x_{3}=0$ with injector flow rates of (a) 0 , (b) 0.78, (c) 0.97, (d) 1.07, (e) 1.25, (f) 1.37, and $(\mathrm{g}) 1.68 \mathrm{~m} \ell / \mathrm{s}$. Dashed lines represent velocity profiles without the injector. The flow rate chosen for the present experiments was $Q=0.97 \mathrm{~m} \ell / \mathrm{s}$.

diameters. As representative of these velocity maps, we present transverse profiles of the mean velocity in figure 13 . The physical presence of the injection tube created a region of relative velocity deficit due to the boundary layer that grew along the tube. At the same time, injection at a velocity higher than the local value created a local momentum surplus within the injected fluid. Our objective was to adjust the injection flow rate so that the momentum surplus of the jet would compensate, as much as possible, for the momentum deficit around the injector, without introducing significant jet effects which would distort the structure of the USF. The value $Q=0.97 \mathrm{~m} \ell / \mathrm{s}$, represented by curve (c) in figure 13, was deemed to be the optimal one and was used for all reported results.

\section{REFERENCES}

Adrian, R.J. \& Westerweel, J. 2011 Particle Image Velocimetry. Cambridge University Press.

AnAnd, M.S. \& Pope, S.B. 1985 Diffusion behind a line source in grid turbulence. In Turbulent Shear Flows 4, pp. 46-61. Springer.

ARYA, S.P. 1999 Air Pollution Meteorology and Dispersion. Oxford University Press, New York.

Batchelor, G.K. 1949 Diffusion in a field of homogeneous turbulence. I. Eulerian analysis. Aust. J. Chem. 2 (4), 437-450.

Borg, A., Bolinder, J. \& Fuchs, L. 2001 Simultaneous velocity and concentration measurements in the near field of a turbulent low-pressure jet by digital particle image velocimetryplanar laser-induced fluorescence. Exp. Fluids 31 (2), 140-152.

Chang, K.-A. \& Cowen, E.A. 2002 Turbulent prandtl number in neutrally buoyant turbulent round jet. J. Eng. Mech.-ASCE 128 (10), 1082-1087.

CorRsin, S. 1974 Limitations of gradient transport models in random walks and in turbulence. Adv. Geophys. p. 25.

Csanady, G.T. 1963 Turbulent diffusion in Lake Huron. J. Fluid Mech. 17 (03), 360-384.

De Souza, F.A., Nguyen, V.D. \& Tavoularis, S. 1995 The structure of highly sheared turbulence. J. Fluid Mech. 303, 155-167.

DeArdorfF, J.W. 1966 The counter-gradient heat flux in the lower atmosphere and in the laboratory. J. Atmos. Sci. 23, 503-506.

van Dop, H. \& Verver, G. 2001 Countergradient transport revisited. J. Atmos. Sci. 58 (15), 2240-2247.

Fukushima, C., Aanen, L. \& Westerweel, J. 2000 Investigation of the mixing process in an 
axisymmetric turbulent jet using PIV and LIF. In Laser Techniques for Fluid Mechanics (ed. R.J. Adrian et al.), pp. 339-356. Springer, Berlin.

Gendron, P.O., Avaltroni, F. \& Wilkinson, K.J. 2008 Diffusion coefficients of several rhodamine derivatives as determined by pulsed field gradient-nuclear magnetic resonance and fluorescence correlation spectroscopy. J. Fluoresc. 18 (6), 1093-1101.

HAY, J.S. \& PASQUILL, F. 1959 Diffusion from a continuous source in relation to the spectrum and scale of turbulence. Adv. Geophys. 6, 345-365.

Karnik, U. \& TAvoularis, S. 1987 Generation and manipulation of uniform shear with the use of screens. Exp. Fluids 5, 247-254.

Karnik, U. \& TAVoularis, S. 1989 Measurements of heat diffusion from a continuous line source in a uniformly sheared turbulent flow. J. Fluid Mech. 202, 233-261.

KARniK, U. \& TAVOularis, S. 1990 Lagrangian correlations and scales in uniformly sheared turbulence. Phys. Fluids A 2 (4), 587-591.

Lemoine, F., Wolff, M. \& Lebouche, M. 1996 Simultaneous concentration and velocity measurements using combined laser-induced fluorescence and laser doppler velocimetry: Application to turbulent transport. Exp. Fluids 20 (5), 319-327.

Lepore, J. \& Mydlarski, L. 2011 Lateral dispersion from a concentrated line source in turbulent channel flow. J. Fluid Mech. 678, 417-450.

Nakamura, I., Sakai, Y., Miyata, M. \& Tsunoda, H. 1986 Diffusion of matter from a continuous point source in uniform mean shear flows (1st report). B. JSME 29 (250), 1141-1148.

O’Neill, P.L., Nicolaides, D., Honnery, D. \& Soria, J. 2004 Autocorrelation functions and the determination of integral length with reference to experimental and numerical data. In Proceedings of the Fifteenth Australasian Fluid Mechanics Conference. University of Sydney.

Paranthö̈n, P., Godard, G., Weiss, F. \& Gonzalez, M. 2004 Counter gradient diffusion vs "counter diffusion" temperature profile? Int. J. Heat Mass Trans 47 (4), 819-825.

Pope, S. 2000 Turbulent Flows. Cambridge University Press.

Prasad, A.K. \& Jensen, K. 1995 Scheimpflug stereocamera for particle image velocimetry in liquid flows. Appl. Opt. 34 (30), 7092-7099.

Rahman, S. \& Webster, D.R. 2005 The effect of bed roughness on scalar fluctuations in turbulent boundary layers. Experiments in Fluids 38 (3), 372-384.

Riley, J.J. \& CoRrsin, S. 1974 The relation of turbulent diffusivities to Lagrangian velocity statistics for the simplest shear flow. J. Geophys. Res. 79 (12), 1768-1771.

Roberts, O.F.T. 1923 The theoretical scattering of smoke in a turbulent atmosphere. Proc. $R$. Soc. Lond. A 104 (728), 640-654.

Roberts, P.J.W. \& Webster, D.R. 2002 Turbulent diffusion. In Environmental Fluid Mechanics: Theories and Applications (ed. H. Shen), pp. 7-45. American Society of Civil Engineers.

Rogers, M.M., Mansour, N.N. \& Reynolds, W.C. 1989 An algebraic model for the turbulent flux of a passive scalar. J. Fluid Mech. 203 (1), 77-101.

De Roode, S.R., Jonker, H.J.J., Duynkerke, P.G. \& Stevens, B. 2004 Countergradient fluxes of conserved variables in the clear convective and stratocumulus-topped boundary layer: The role of the entrainment flux. Bound-Lay Meteorol 112 (1), 179-196.

Sreenivasan, K.R., Tavoularis, S. \& Corrsin, S. 1982 A test of gradient transport and its generalizations. In Turbulent Shear Flows 3 (ed. Bradbury, Durst, Launder, Schmidt \& Whitelaw), pp. 96-112. Springer Berlin Heidelberg.

Stapountzis, H., Sawford, B.L., Hunt, J.C.R. \& Britter, R.E. 1986 Structure of the temperature field downwind of a line source in grid turbulence. J. Fluid Mech 165, 401424.

Sutton, O.G. 1932 A theory of eddy diffusion in the atmosphere. Proc. R. Soc. Lond. A 135 (826), 143-165.

TAvoularis, S. \& Corrsin, S. 1981 Experiments in nearly homogeneous turbulent shear flow with a uniform mean temperature gradient. Part 1. J. Fluid Mech. 104, 311-347.

Tavoularis, S. \& Corrsin, S. 1985 Effects of shear on the turbulent diffusivity tensor. Int. J. Heat Mass Trans 28 (1), 265-276. 
TAYlor, G.I. 1921 Diffusion by continuous movements. Proc. London Math. Soc. 20 (2), 196211.

VAnderwel, C. \& Tavoularis, S. 2011 Coherent structures in uniformly sheared turbulent flow. J. Fluid Mech. 689, 434-464.

VANDERWEL, C. \& TAVOularis, S. $2014 a$ On the accuracy of PLIF measurements in slender plumes. Submitted to Exp. Fluids.

VAnderwel, C. \& Tavoularis, S. $2014 b$ Relative dispersion of a passive scalar plume in turbulent shear flow. Phys. Rev. E 89 (4), 041005(R).

Veeravalli, S. \& WARhaft, Z. 1990 Thermal dispersion from a line source in the shearless turbulence mixing layer. J. Fluid Mech 216, 35-70.

WARHAFt, Z. 1984 The interference of thermal fields from line sources in grid turbulence. $J$. Fluid Mech. 144, 363-387.

Webster, D.R., Rahman, S. \& Dasi, L.P. 2003 Laser-induced fluorescence measurements of a turbulent plume. J. Eng. Mech.-ASCE 129, 1130-1137.

Webster, D.R., Roberts, P.J.W. \& RA'AD, L. 2001 Simultaneous DPTV/PLIF measurements of a turbulent jet. Exp. Fluids 30, 65-72.

Wilson, D.J., Robins, A.G. \& FACKrell, J.E. 1985 Intermittency and conditionally-averaged concentration fluctuation statistics in plumes. Atmos. Environ. 19 (7), 1053-1064.

Würth, C., González, M.G., Niessner, R., Panne, U., Haisch, C. \& Genger, U.R. 2012 Determination of the absolute fluorescence quantum yield of Rhodamine 6G with optical and photoacoustic methods-providing the basis for fluorescence quantum yield standards. Talanta 90, 30-37.

Younis, B.A., Speziale, C.G. \& Clark, T.T. 2005 A rational model for the turbulent scalar fluxes. Proc. R. Soc. A 461, 575-594. 